\title{
Reduced basis approximation and $a$ posteriori error estimation for parametrized parabolic PDEs; Application to real-time Bayesian parameter estimation
}

\author{
N.C. Nguyen, G. Rozza, D.B.P. Huynh, and A.T. Patera \\ Massachusetts Institute of Technology, Department of Mechanical Engineering, \\ Room 3-264, 77 Massachusetts Avenue, Cambridge MA, 02142-4307, USA.
}

\subsection{Introduction}

In this chapter we consider reduced basis approximation and a posteriori error estimation for linear functional outputs of affinely parametrized linear and non-linear parabolic partial differential equations. The essential ingredients are Galerkin projection onto a low-dimensional space associated with a smooth "parametric manifold" dimension reduction; efficient and effective Greedy and POD-Greedy sampling methods for identification of optimal and numerically stable approximations — rapid convergence; rigorous and sharp a posteriori error bounds (and associated stability factors) for the linear-functional outputs of interest — certainty; and Offline-Online computational decomposition strategies - minimum marginal cost for high performance in the real-time/embedded (e.g., parameter estimation, control) and many-query (e.g., design optimization, uncertainty quantification Boyaval et al. (2008), multi-scale Boyaval (2008); Nguyen (2008b)) contexts.

In this paper we first present reduced basis approximation and a posteriori error estimation Prud'homme et al. (2002); Rozza et al. (to appear 2008) for general linear parabolic equations - building on Grepl and Patera (2005); Haasdonk and Ohlberger (2008) — and subsequently for a nonlinear parabolic equation, the incompressible Navier-Stokes equations — building on Nguyen et al. (2008). We then present results for the application of our (parabolic) reduced basis methods to Bayesian parameter estimation: detection and characterization of a delamination crack by transient thermal analysis Grepl (2005); Starnes (2002).

Correspondence to cuongng@mit.edu (N.C. Nguyen), rozza@mit .edu (G. Rozza), patera@mit.edu (A.T. Patera).

Computational Methods for Large-Scale Inverse Problems and Quantification of Uncertainty. Edited by L. Biegler, G. Biros, O. Ghattas, M. Heinkenschloss, D. Keyes, B. Mallick, L. Tenorio, B. van Bloemen Waanders, K. Willcox. We would like to thank Dr. Paul Fischer of Argonne National Laboratory and Prof. Yvon Maday of University Paris VI for helpful discussions. This work was supported by AFOSR Grant FA9550-07-1-0425 and the Singapore-MIT Alliance.

(c) 2001 John Wiley \& Sons, Ltd 


\subsection{Linear Parabolic Equations}

\subsubsection{Reduced basis approximation}

We first introduce several notations required for the remainder of the chapter. Our parameter domain, a closed subset of $\mathbb{R}^{P}$, shall be denoted $\mathcal{D}$; a typical parameter value - a $P$-tuple in $\mathcal{D}-$ shall be denoted $\mu$. Our time domain shall be denoted by $I=\left[0, t_{f}\right]$ with $t_{f}$ the final time. Our physical domain in $d$ space dimensions shall be denoted $\Omega$ with boundary $\partial \Omega$; a typical point in $\Omega$ shall be denoted $x=\left(x_{1}, \ldots, x_{d}\right)$. We can then define the function space $X=X(\Omega)$ such that $\left(H_{0}^{1}(\Omega)\right)^{\mathcal{V}} \subset X \subset\left(H^{1}(\Omega)\right)^{\mathcal{V}}$; here $H^{1}(\Omega)=\left\{v \mid v \in L^{2}(\Omega), \nabla v \in\left(L^{2}(\Omega)\right)^{d}\right\}$, $H_{0}^{1}(\Omega)=\left\{v \in H^{1}(\Omega) \mid v_{\partial \Omega}=0\right\}, L^{2}(\Omega)$ is the space of square integrable functions over $\Omega$, and $\mathcal{V}=1$ (respectively, $d$ ) for scalar (respectively, vector) problems. We denote by $(\cdot, \cdot)_{X}$ the inner product associated with the Hilbert space $X$; this inner product in turn induces a norm $\|\cdot\|_{X}=\sqrt{(\cdot, \cdot)_{X}}$ equivalent to the usual $\left(H^{1}(\Omega)\right)^{\mathcal{V}}$ norm. Similarly, we denote by $(\cdot, \cdot)$ and $\|\cdot\|$ the $L^{2}(\Omega)$ inner product and induced norm, respectively.

Given $\mu \in \mathcal{D}$, we find $u(\mu) \in C^{0}\left(I ; L^{2}(\Omega)\right) \cap L^{2}(I ; X)$ such that

$$
m\left(u_{t}(t ; \mu), v ; \mu\right)+a(u(t ; \mu), v ; \mu)=g(t) f(v), \quad \forall v \in X, \quad \forall t \in I,
$$

subject to initial condition $u(t=0 ; \mu)=u_{0} \in L^{2}(\Omega)$. We then evaluate our output as $s(t ; \mu)=\ell(u(t ; \mu)), \forall t \in I$. Note $g(t) \in L^{2}(I)$ is our control function. Our bilinear forms $a$ and $m$ are assumed to be continuous over $X$ and $L^{2}(\Omega)$, respectively. We further suppose coercivity: for all $\mu \in \mathcal{D}$,

$$
\alpha(\mu)=\inf _{w \in X} \frac{a(w, w ; \mu)}{\|w\|_{X}^{2}}
$$

is strictly positive. Finally, we assume that our bilinear forms are "affine in parameter": for some finite $Q_{a}$ and $Q_{m}, a$ and $m$ may be expressed as

$$
a(w, v ; \mu)=\sum_{q=1}^{Q_{a}} \Theta_{a}^{q}(\mu) a^{q}(w, v), \quad m(w, v ; \mu)=\sum_{q^{\prime}=1}^{Q_{m}} \Theta_{m}^{q^{\prime}}(\mu) m^{q^{\prime}}(w, v)
$$

for given parameter-dependent functions $\Theta_{a}^{q}, 1 \leq q \leq Q_{a}, \Theta_{m}^{q^{\prime}}, 1 \leq q^{\prime} \leq Q_{m}$, and continuous parameter-independent bilinear forms $a^{q}, 1 \leq q \leq Q_{a}, m^{q^{\prime}}, 1 \leq q^{\prime} \leq Q_{m}$. (The assumption of affine parameter dependence may be relaxed, see Barrault et al. (2004), however the error estimates — as developed below in the affine case — are no longer rigorously bounds in all cases.) Finally, $f$ and $\ell$ are linear continuous functionals over $X$ and $L^{2}(\Omega)$, respectively; we assume - solely for simplicity of exposition - that $f$ and $\ell$ are independent of $\mu$.

It is important to note that $\Omega$ is not a function of the parameter $\mu$. We implicitly assume that $\Omega$ is a parameterindependent reference domain: all geometric parametric dependence is reflected - through the usual transformation procedures - in the functions $\Theta_{a, m}^{q}(\mu), 1 \leq q \leq Q_{a, m}$. We refer to Rozza et al. (to appear 2008) for automated piecewise-affine mapping procedures $\Omega^{\text {orig }}(\mu) \rightarrow \Omega-$ here $\Omega^{\text {orig }}(\mu)$ is the "original" parameterdependent domain and $\Omega$ is our parameter-independent reference domain — that yield, for rather general geometric parameter dependence, the requisite affine structure (2).

We next introduce the finite difference in time and finite element (FE) in space discretization of this parabolic problem Quarteroni and Valli (1997). We first divide the time interval $I$ into $K$ subintervals of equal length $\Delta t=t_{f} / K$ and define $t^{k} \equiv k \Delta t, 0 \leq k \leq K$. We then define the finite element approximation space $X^{\mathcal{N}} \subset X$ of dimension $\mathcal{N}$. Now, given $\mu \in \mathcal{D}$, we look for $u^{\mathcal{N} k}(\mu) \in X, 0 \leq k \leq K$, such that

$$
\frac{1}{\Delta t} m\left(u^{\mathcal{N} k}(\mu)-u^{\mathcal{N} k-1}(\mu), v ; \mu\right)+a\left(u^{k}(\mu), v ; \mu\right)=g\left(t^{k}\right) f(v), \quad \forall v \in X, 1 \leq k \leq K,
$$

subject to initial condition $\left(u^{\mathcal{N} 0}, v\right)=\left(u_{0}, v\right), \forall v \in X^{\mathcal{N}}$. We then evaluate the output: for $0 \leq k \leq K$,

$$
s^{\mathcal{N} k}(\mu)=\ell\left(u^{\mathcal{N} k}(\mu)\right) .
$$

We shall sometimes denote $u^{\mathcal{N} k}(\mu)$ as $u^{\mathcal{N}}\left(t^{k} ; \mu\right)$ and $s^{\mathcal{N} k}(\mu)$ as $s^{\mathcal{N}}\left(t^{k} ; \mu\right)$ to more clearly identify the discrete time levels. Equation (3) — Euler-Backward Galerkin discretization of (1) — shall be our point of departure: we shall presume that $\Delta t$ is sufficiently small and $\mathcal{N}$ is sufficiently large such that $u^{\mathcal{N}}\left(t^{k} ; \mu\right)$ and $s^{\mathcal{N}}\left(t^{k} ; \mu\right)$ are effectively indistinguishable from $u\left(t^{k} ; \mu\right)$ and $s\left(t^{k} ; \mu\right)$, respectively. (The development readily extends to Crank-Nicolson discretization; for purposes of exposition, we consider the simple Euler Backward approach.) 
Finally, we introduce the reduced basis (RB) approximation Almroth et al. (1978); Fink and Rheinboldt (1983); Noor and Peters (1980); Porsching (1985). Given a set of mutually $(\cdot, \cdot)_{X}$-orthonormal basis functions $\xi_{n} \in$ $X^{\mathcal{N}}, 1 \leq n \leq N_{\max }$, the RB spaces are given by

$$
X_{N} \equiv \operatorname{span}\left\{\xi_{n}, 1 \leq n \leq N\right\}, \quad 1 \leq N \leq N_{\max }
$$

In actual practice (see Section 0.2.3), the spaces $X_{N} \in X^{\mathcal{N}}$ will be generated by a POD-Greedy sampling procedure which combines spatial snapshots in time and parameter $-u^{\mathcal{N} k}(\mu)-$ in an optimal fashion; for our present purposes, however, $X_{N}$ can in fact represent any sequence of (low-dimensional) hierarchical approximation spaces Rozza et al. (to appear 2008). Given $\mu \in \mathcal{D}$, we now look for $u_{N}^{k}(\mu) \in X_{N}, 0 \leq k \leq K$, such that

$$
\frac{1}{\Delta t} m\left(u_{N}^{k}(\mu)-u_{N}^{k-1}(\mu), v ; \mu\right)+a\left(u_{N}^{k}(\mu), v ; \mu\right)=g\left(t^{k}\right) f(v), \quad \forall v \in X_{N}, 1 \leq k \leq K,
$$

subject to $\left(u_{N}^{0}(\mu), v\right)=\left(u^{\mathcal{N} 0}, v\right), \forall v \in X_{N}$. We then evaluate the associated output: for $0 \leq k \leq K$,

$$
s_{N}^{k}(\mu)=\ell\left(u_{N}^{k}(\mu)\right)
$$

We shall sometimes denote $u_{N}^{k}(\mu)$ as $u_{N}\left(t^{k} ; \mu\right)$ and $s_{N}^{k}(\mu)$ as $s_{N}\left(t^{k} ; \mu\right)$ to more clearly identify the discrete time levels. (Note that in fact all the RB quantities should bear a $\mathcal{N}-X_{N}^{\mathcal{N}}, u_{N}^{\mathcal{N} k}(\mu), s_{N}^{\mathcal{N} k}(\mu)-$ since the RB approximation is defined in terms of a particular truth discretization; however, for clarity of exposition, we shall typically suppress the "truth" superscript.)

The goal of the RB approximation is simple: dimension reduction $-N \ll \mathcal{N}-$ and associated computational economies. (Online) RB evaluation is typically several orders of magnitude less expensive than the classical finite element approach Prud'homme et al. (2002); Rozza et al. (to appear 2008).

\subsubsection{A posteriori error estimation}

Crucial to the general area of model reduction is not just the reduced-order approximation but even more importantly rigorous, sharp, and inexpensive a posteriori error bounds. To construct the a posteriori error bounds for the RB approximation, we need two ingredients. The first ingredient is the dual norm of the residual

$$
\varepsilon_{N}\left(t^{k} ; \mu\right)=\sup _{v \in X^{\mathcal{N}}} \frac{r_{N}\left(v ; t^{k} ; \mu\right)}{\|v\|_{X}}, \quad 1 \leq k \leq K,
$$

where $r_{N}\left(v ; t^{k} ; \mu\right)$ is the residual associated with the RB approximation (6)

$$
r_{N}\left(v ; t^{k} ; \mu\right)=g\left(t^{k}\right) f(v)-\frac{1}{\Delta t} m\left(u_{N}^{k}(\mu)-u_{N}^{k-1}(\mu), v ; \mu\right)-a\left(u_{N}^{k}(\mu), v ; \mu\right), \quad \forall v \in X^{\mathcal{N}}, \quad 1 \leq k \leq K .
$$

The second ingredient is a lower bound $\alpha_{\mathrm{LB}}^{\mathcal{N}}(\mu) \leq \alpha^{\mathcal{N}}(\mu), \forall \mu \in \mathcal{D}$, for the stability constant $\alpha^{\mathcal{N}}(\mu)$ defined as

$$
\alpha^{\mathcal{N}}(\mu)=\inf _{v \in X^{\mathcal{N}}} \frac{a(v, v ; \mu)}{\|v\|_{X}^{2}}, \quad \forall \mu \in \mathcal{D} \text {. }
$$

Note that since $X^{\mathcal{N}} \subset X, \alpha^{\mathcal{N}}(\mu) \geq \alpha(\mu)>0, \forall \mu \in \mathcal{D}$; we further assume that $\alpha_{\text {LB }}^{\mathcal{N}}(\mu)>0, \forall \mu \in \mathcal{D}$.

We can now define our error bounds in terms of the dual norm of the residual and the lower bound for the stability constant. In particular, it can readily be proven Grepl and Patera (2005); Haasdonk and Ohlberger (2008); Nguyen et al. (2008) that for all $\mu \in \mathcal{D}$ and all $N$,

$$
\left\|u^{\mathcal{N} k}(\mu)-u_{N}^{k}(\mu)\right\| \leq \Delta_{N}^{k}(\mu), \quad\left|s^{\mathcal{N} k}(\mu)-s_{N}^{k}(\mu)\right| \leq \Delta_{N}^{s k}(\mu), \quad 1 \leq k \leq K,
$$

where $\Delta_{N}^{k}(\mu) \equiv \Delta_{N}\left(t^{k} ; \mu\right)$ and $\Delta_{N}^{s k}(\mu) \equiv \Delta_{N}^{s}\left(t^{k} ; \mu\right)$ are given by

$$
\begin{aligned}
\Delta_{N}^{k}(\mu) & \equiv \sqrt{\frac{\frac{\Delta t}{\alpha_{\mathrm{LB}}^{\mathcal{N}}(\mu)} \sum_{k^{\prime}=1}^{k}\left(\varepsilon_{N}^{2}\left(t^{k^{\prime}} ; \mu\right)\left(1+\Delta t \alpha_{\mathrm{LB}}^{\mathcal{N}}(\mu)\right)^{k^{\prime}-1}\right)}{\left(1+\Delta t \alpha_{\mathrm{LB}}^{\mathcal{N}}(\mu)\right)^{k}}}, \\
\Delta_{N}^{s k}(\mu) & \equiv\left(\sup _{v \in X^{\mathcal{N}}} \frac{\ell(v)}{\|v\|}\right) \Delta_{N}^{k}(\mu) .
\end{aligned}
$$


(We assume for simplicity that $u^{\mathcal{N} 0} \in X_{N}$; otherwise there will be an additional contribution to $\Delta_{N}^{k}(\mu)$ ). Note again that the RB error is measured relative to the finite element "truth."

It should be clear that our error bound for the output is rather crude. We may pursue primal-dual RB approximations Grepl and Patera (2005); Pierce and Giles (2000); Rozza et al. (to appear 2008) that provide both more rapid convergence of the output and also more robust (sharper) estimation of the output error. However, in cases in which many outputs are of interest, for example inverse problems, the primal-only approach described above can be more efficient and also more adaptive - efficiently expanded to include additional outputs.

\subsubsection{Offline-Online computational approach}

\section{Construction-Evaluation decomposition}

The affine representation (2) permits a "Construction-Evaluation" decomposition Balmes (1996); Machiels et al. (2000); Prud'homme et al. (2002) of computational effort that greatly reduces the marginal cost — relevant in the real-time and many-query contexts — of both the RB output evaluation, (7), and the associated error bound, (11). The expensive Construction stage, performed once, provides the foundation for the subsequent very inexpensive Evaluation stage, performed many times for each new desired $\mu \in \mathcal{D}$. We first consider the Construction-Evaluation decomposition for the output and then address the error bound.

We represent $u_{N}^{k}(\mu)$ as $u_{N}^{k}(\mu)=\sum_{n=1}^{N} \omega_{N n}^{k}(\mu) \xi_{n}$, where we recall that the $\xi_{j}, 1 \leq j \leq N$, are the basis functions for our RB space $X_{N}$. We may then evaluate the RB output as

$$
s_{N}^{k}(\mu)=\mathcal{L}_{N}^{T} \omega_{N}(\mu), \quad 1 \leq k \leq K
$$

where $\mathcal{L}_{N n}=\ell\left(\xi_{n}\right), 1 \leq n \leq N$. To find the $\omega_{N j}^{k}(\mu), 1 \leq j \leq N, 1 \leq k \leq K$, we insert $u_{N}^{k}(\mu)=\sum_{n=1}^{N} \omega_{N n}^{k}(\mu) \xi_{n}$, $u_{N}^{k-1}(\mu)=\sum_{n=1}^{N} \omega_{N n}^{k-1}(\mu) \xi_{n}$, and $v=\xi_{m}$ in (6) to obtain the discrete system

$$
\left(\mathcal{M}_{N}(\mu)+\Delta t \mathcal{A}_{N}(\mu)\right) \omega_{N}^{k}(\mu)=\Delta t g\left(t^{k}\right) \mathcal{F}_{N}+\mathcal{M}_{N}(\mu) \omega_{N}^{k-1}(\mu), \quad 1 \leq k \leq K,
$$

where $\mathcal{A}_{N}(\mu) \in \mathbb{R}^{N \times N}, \mathcal{M}_{N}(\mu) \in \mathbb{R}^{N \times N}$, and $\mathcal{F}_{N}(\mu) \in \mathbb{R}^{N}$ are given by $\mathcal{A}_{N m, n}=a\left(\xi_{n}, \xi_{m} ; \mu\right), \mathcal{M}_{N m, n}=$ $m\left(\xi_{n}, \xi_{m} ; \mu\right), 1 \leq m, n \leq N$, and $\mathcal{F}_{N n}=f\left(\xi_{n}\right), 1 \leq n \leq N$, respectively. We note that $\mathcal{A}_{N}(\mu)$ and $\mathcal{M}_{N}(\mu)$ can be expressed, thanks to (2), as

$$
\mathcal{A}_{N}(\mu)=\sum_{q=1}^{Q_{a}} \Theta_{a}^{q}(\mu) \mathcal{A}_{N}^{q}, \quad \mathcal{M}_{N}(\mu)=\sum_{q^{\prime}=1}^{Q_{m}} \Theta_{m}^{q^{\prime}}(\mu) \mathcal{M}_{N}^{q^{\prime}},
$$

where the $\mathcal{A}_{N m, n}^{q} \equiv a^{q}\left(\xi_{n}, \xi_{m}\right), \mathcal{M}_{N m, n}^{q^{\prime}} \equiv m^{q^{\prime}}\left(\xi_{n}, \xi_{m}\right), 1 \leq m, n \leq N, 1 \leq q \leq Q_{a}, 1 \leq q^{\prime} \leq Q_{m}$, are parameterindependent. We can now readily identify the Construction-Evaluation decomposition.

In the Construction stage we first form and store the time-independent and $\mu$-independent matrices/vectors $\mathcal{A}_{N_{\max } i j}^{q}, \mathcal{M}_{N_{\max } i j}^{q^{\prime}}, \mathcal{F}_{N_{\max } i}$, and $\mathcal{L}_{N_{\max } i}, 1 \leq i, j \leq N_{\max }, 1 \leq q \leq Q_{a}, 1 \leq q^{\prime} \leq Q_{m}$. The operation count in the Construction stage of course depends on $\mathcal{N}$ - even once the $\xi_{i}, 1 \leq i \leq N_{\max }$, are known (obtained by the sampling procedure of the next section), it remains to compute $O\left(N_{\max }^{2}\right)$ finite element quadratures over the $O(\mathcal{N})$ triangulation. Note that, thanks to the hierarchical nature of the RB spaces, the stiffness matrices/vectors $\mathcal{A}_{N i j}^{q}, \mathcal{M}_{N i j}^{q^{\prime}}, \mathcal{F}_{N i}$, and $\mathcal{L}_{N i}, 1 \leq i, j \leq N$, for any $N \leq N_{\text {max }}$ can be extracted as principal subarrays of the corresponding $N_{\max }$ quantities. (For nonhierarchical RB spaces the storage requirements are much higher.)

In the Evaluation stage, we first form the left-hand side of (13) in $O\left(\left(Q_{a}+Q_{m}\right) N^{2}\right)$ operations; we then invert the resulting $N \times N$ matrix in $O\left(N^{3}\right)$ operations (in general, we must anticipate that the RB matrices will be dense); finally, we compute $\omega_{N j}^{k}, 1 \leq j \leq N, 1 \leq k \leq K$, in $O\left(K N^{2}\right)$ operations by matrix-vector multiplication. Once the $\omega_{N j}^{k}, 1 \leq j \leq N, 1 \leq k \leq K$, are obtained - $O\left(\left(Q_{a}+Q_{m}+N+K\right) N^{2}\right)$ operations in total - we evaluate our output from (12) in $O(N K)$ operations. The storage and operation count in the Evaluation phase is clearly independent of $\mathcal{N}$, and we can thus anticipate - presuming $N \ll \mathcal{N}$ - very rapid RB response in the real-time and many-query contexts.

The Construction-Evaluation procedure for the output error bound is a bit more involved. There are three components to this bound: the dual norm of $\ell$ (readily computed, once, in the Construction phase); the lower bound for the coercivity constant, $\alpha_{\mathrm{LB}}(\mu)$, which is computed by the Successive Constraint Method (SCM) as described in Huynh et al. (2007); Rozza et al. (to appear 2008), and Section 0.3.3 of the current paper; and the dual norm of the residual $\varepsilon_{N}\left(t^{k} ; \mu\right)$. We consider here the Construction-Evaluation decomposition for the dual 
norm of the residual Grepl and Patera (2005). We first invoke duality, our RB expansion, the affine parametric dependence of $a$ and $m$, and linear superposition to express

$$
\begin{aligned}
\varepsilon_{N}^{2}\left(t^{k} ; \mu\right)= & \mathcal{Q}_{N}^{f f}+\sum_{n=1}^{N}\left(\sum_{q=1}^{Q_{a}} \Theta_{a}^{q}(\mu) \omega_{N n}^{k}(\mu) \mathcal{Q}_{N n q}^{f a}+\frac{1}{\Delta t} \sum_{q^{\prime}=1}^{Q_{m}} \Theta_{m}^{q^{\prime}}(\mu)\left(\omega_{N n}^{k}(\mu)-\omega_{N n}^{k-1}(\mu)\right) \mathcal{Q}_{N n q^{\prime}}^{f m}\right) \\
& +\sum_{n, n^{\prime}=1}^{N, N}\left(\sum_{q, q^{\prime}=1}^{Q_{a}, Q_{a}} \Theta_{a}^{q}(\mu) \Theta_{a}^{q^{\prime}}(\mu) \omega_{N n}^{k}(\mu) \omega_{N n^{\prime}}^{k}(\mu) \mathcal{Q}_{N n n^{\prime} q q^{\prime}}^{a a}\right. \\
& +\frac{1}{(\Delta t)^{2}} \sum_{q=1}^{Q_{m}} \sum_{q^{\prime}=1}^{Q_{m}} \Theta_{m}^{q}(\mu) \Theta_{m}^{q^{\prime}}(\mu)\left(\omega_{N n}^{k}(\mu)-\omega_{N n}^{k-1}(\mu)\right)\left(\omega_{N n^{\prime}}^{k}(\mu)-\omega_{N n^{\prime}}^{k-1}(\mu)\right) \mathcal{Q}_{N n n^{\prime} q q^{\prime}}^{m m} \\
& \left.+\frac{1}{\Delta t} \sum_{q=1}^{Q_{a}} \sum_{q^{\prime}=1}^{Q_{m}} \Theta_{a}^{q}(\mu) \Theta_{m}^{q^{\prime}}(\mu) \omega_{N n}^{k}(\mu)\left(\omega_{N n^{\prime}}^{k}(\mu)-\omega_{N n^{\prime}}^{k-1}(\mu)\right) \mathcal{Q}_{N n n^{\prime} q q^{\prime}}^{a m}\right), \quad 1 \leq k \leq K
\end{aligned}
$$

where $\mathcal{Q}_{N}^{f f}=\left(z^{f}, z^{f}\right)_{X}, \mathcal{Q}_{N n q}^{f a}=2\left(z_{n q}^{a}, z^{f}\right)_{X}, 1 \leq q \leq Q_{a}, 1 \leq n \leq N, \mathcal{Q}_{N n q}^{f m}=2\left(z_{n q}^{m}, z^{f}\right)_{X}, 1 \leq q \leq Q_{m}$, $1 \leq n \leq N, \mathcal{Q}_{N n n^{\prime} q q^{\prime}}^{a a}=\left(z_{n q}^{a}, z_{n^{\prime} q^{\prime}}^{a}\right)_{X}, 1 \leq q, q^{\prime} \leq Q_{a}, 1 \leq n, n^{\prime} \leq N, \mathcal{Q}_{N n n^{\prime} q q^{\prime}}^{a m}=2\left(z_{n q}^{a}, z_{n^{\prime} q^{\prime}}^{m}\right)_{X}, 1 \leq q \leq$ $Q_{a}, 1 \leq q^{\prime} \leq Q_{m}, 1 \leq n, n^{\prime} \leq N$, and $\mathcal{Q}_{N n n^{\prime} q q^{\prime}}^{m m}=\left(z_{n q}^{m}, z_{n^{\prime} q^{\prime}}^{m}\right)_{X}, 1 \leq q, q^{\prime} \leq Q_{m}, 1 \leq n, n^{\prime} \leq N$. Here the $z^{f}, z_{n q}^{a}, z_{n q^{\prime}}^{m}$ are solutions to time-independent and $\mu$-independent "Poisson" problems: $\left(z^{f}, v\right)_{X}=f(v), \forall v \in$ $X^{\mathcal{N}},\left(z_{n q}^{a}, v\right)_{X}=-a^{q}\left(\xi_{n}, v\right), \forall v \in X^{\mathcal{N}}, 1 \leq n \leq N, 1 \leq q \leq Q_{a}$, and $\left(z_{n q^{\prime}}^{m}, v\right)_{X}=-m^{q^{\prime}}\left(\xi_{n}, v\right), \forall v \in X^{\mathcal{N}}$, $1 \leq n \leq N, 1 \leq q^{\prime} \leq Q_{m}$

The Construction-Evaluation decomposition is now clear. In the construction stage - parameter independent, and performed only once - we find $z^{f}, z^{a}, z^{m}$, and the inner products $\mathcal{Q}_{N_{\max }}^{f f}, \mathcal{Q}_{N_{\max }}^{f a}, \mathcal{Q}_{N_{\max } m}^{f m}, \mathcal{Q}_{N_{\max }}^{a a}, \mathcal{Q}_{N_{\max } m}^{m m}$, and $\mathcal{Q}_{N_{\max }}^{a m}$ at (considerable) computational cost $O\left(Q_{a}^{\cdot} Q_{m}^{\cdot} N_{\max }^{\cdot} \mathcal{N}^{\cdot}\right)$. We note again that the inner products $\mathcal{Q}_{N}^{f f}$, $\mathcal{Q}_{N}^{f a}, \mathcal{Q}_{N}^{f m}, \mathcal{Q}_{N}^{a a}, \mathcal{Q}_{N}^{m m}$, and $\mathcal{Q}_{N}^{a m}$ for any $N \leq N_{\max }$ can be extracted as principal subarrays of the corresponding $N_{\max }$ quantities. In the Evaluation stage — parameter dependent, and performed many times — we simply perform the sum (15) from the stored inner products in $O\left(\left(1+Q_{m} N+Q_{a} N\right)^{2}\right)$ operations per time step and hence $O\left(\left(1+Q_{m} N+Q_{a} N\right)^{2} K\right)$ operations in total. The crucial point, again, is that the cost and storage in the Evaluation phase - the marginal cost for each new value of $\mu-$ is independent of $\mathcal{N}$ : thus we can not only evaluate our output prediction but also our rigorous output error bound very rapidly in the parametrically interesting contexts of real-time or many-query investigation. In short, we inherit the high fidelity and certainty of the FE approximation but at the low cost of a reduced-order model.

\section{POD-Greedy sampling strategy}

Our sampling method (see also Nguyen et al. (2008)) follows the proposal in Haasdonk and Ohlberger (2008): we combine the POD (Proper Orthogonal Decomposition) in $t^{k}$ - to capture the causality associated with our evolution equation - with the Greedy procedure in $\mu$ Grepl and Patera (2005); Rozza et al. (to appear 2008); Veroy et al. (2003b) - to treat efficiently the higher dimensions and more extensive ranges of parameter variation. To begin, we summarize the well-known optimality property of the POD as described in Kunisch and Volkwein (2002). Given $J$ elements of $X^{\mathcal{N}}, w_{j} \in X^{\mathcal{N}}, 1 \leq j \leq J, \operatorname{POD}\left(\left\{w_{1}, \ldots, w_{J}\right\}, M\right)$ returns $M(\cdot, \cdot)_{X}$-orthonormal functions $\left\{\chi_{m}, 1 \leq m \leq M\right\}$ such that the space $\mathcal{P}_{M}=\operatorname{span}\left\{\chi_{m}, 1 \leq m \leq M\right\}$ is optimal in the sense that

$$
\mathcal{P}_{M}=\arg \inf _{Y_{M} \subset \operatorname{span}\left\{w_{j}, 1 \leq j \leq J\right\}}\left(\frac{1}{J} \sum_{j=1}^{J} \inf _{v \in Y_{M}}\left\|w_{j}-v\right\|_{X}^{2}\right)^{1 / 2}
$$

where $Y_{M}$ denotes an $M$-dimensional linear space. 
To initiate the POD-Greedy sampling procedure we must specify a very large (exhaustive) "train" sample of $n_{\text {train }}$ points in $\mathcal{D}, \Xi_{\text {train }}$, and an initial (say, random) sample $S^{*}=\left\{\mu_{0}^{*}\right\}$. The algorithm is then given by

$$
\begin{aligned}
& \text { Set } \mathcal{Z}=\emptyset, \mu^{*}=\mu_{0}^{*} ; \\
& \text { While } \quad N \leq N_{\max , 0} \\
& \qquad\left\{\chi_{m}, 1 \leq m \leq M_{1}\right\}=\operatorname{POD}\left(\left\{u^{\mathcal{N}}\left(t^{k}, \mu^{*}\right), 1 \leq k \leq K\right\}, M_{1}\right) ; \\
& \quad \mathcal{Z} \leftarrow\left\{\mathcal{Z},\left\{\chi_{m}, 1 \leq m \leq M_{1}\right\}\right\} ; \\
& \quad N \leftarrow N+M_{2} ; \\
& \quad\left\{\xi_{n}, 1 \leq n \leq N\right\}=\operatorname{POD}(\mathcal{Z}, N) ; \\
& \quad X_{N}=\operatorname{span}\left\{\xi_{n}, 1 \leq n \leq N\right\} ; \\
& \quad \mu^{*}=\arg \max { }_{\mu \in \Xi_{\operatorname{train}}} \Delta_{N}\left(t^{K}=t_{f} ; \mu\right) \\
& \text { end. } \\
& \text { Set } X_{N}=\left\{S^{*}, \mu^{*}\right\} ; \\
&
\end{aligned}
$$

In actual practice, we typically exit the POD-Greedy sampling procedure at $N=N_{\max } \leq N_{\max , 0}$ for which a prescribed error tolerance is satisfied: to wit, we define

$$
\epsilon_{N, \text { max }}^{*}=\max _{\mu \in \Xi_{\text {train }}} \Delta_{N}\left(t^{K} ; \mu\right),
$$

and terminate when $\epsilon_{N, \max }^{*} \leq \epsilon_{\mathrm{tol}}^{*}$. Note, by virtue of the final re-definition, the POD-Greedy generates hierarchical spaces $X_{N}, 1 \leq N \leq N_{\max }$, which is computationally very advantageous.

There are two "tuning" variables in the POD-Greedy procedure, $M_{1}$ and $M_{2}$. We choose $M_{1}$ to satisfy an internal POD error criterion based on the usual sum of eigenvalues and $\epsilon_{\mathrm{tol}}^{*}$; we choose $M_{2} \leq M_{1}$ to minimize duplication in the RB space. It is important to note that the POD-Greedy method readily accommodates a repeat $\mu^{*}$ in successive Greedy cycles - new information will always be available and old information rejected; in contrast, a pure Greedy approach in both $t$ and $\mu$ Grepl and Patera (2005), though often generating good spaces, can "stall." Furthermore, since the POD is conducted in only one (time) dimension - with the Greedy addressing the remaining (parameter) dimensions - the procedure remains computationally feasible even for large parameter domains and very extensive parameter train samples (and in particular in higher parameter dimensions). We now discuss the computational aspects in slightly more detail.

The crucial point to note is that the operation count for the POD-Greedy algorithm is additive and not multiplicative in $n_{\text {train }}$ and $\mathcal{N}$. In particular, in searching for the next parameter value $\mu^{*}$, we invoke the ConstructionEvaluation decomposition to inexpensively calculate the a posteriori error bound at the $n_{\text {train }}$ candidate parameter values. In contrast, in a pure POD approach, we would need to evaluate the finite element "truth" solution at the $n_{\text {train }}$ candidate parameter values. (Of course, much of the computational economies are due not to the Greedy per se, but rather to the accommodation within the Greedy of the inexpensive error bounds.) As a result, in the PODGreedy approach we can take $n_{\text {train }}$ relatively large: we can thus anticipate RB spaces and approximations that provide rapid convergence uniformly over the entire parameter domain. (More sophisticated and hence efficient search algorithms can also be exploited in the Greedy context, for example Bui-Thanh et al. (2007).)

We pursue the POD-Greedy sampling procedure - which involves both the Construction and Evaluation phases - in an Offline stage. Then, in the Online stage, we invoke only the very inexpensive Evaluation phase: $\mu \rightarrow s_{N}^{k}(\mu), \Delta_{N}^{k}(\mu), 1 \leq k \leq K$. Thus either in the real-time context or the many-query context - in which the Offline stage is unimportant and amortized, respectively — the RB approach will be very competitive. Note also in the POD-Greedy procedure we choose for $g(t)$ the impulse function; the resulting RB space will thus have good approximation properties for any $g(t)-g(t)$ can be specified in the Online stage.

\subsection{Nonlinear Parabolic Equations}

\subsubsection{Reduced basis approximation}

We consider here the extension of the RB methods and associated a posteriori error estimators to quadratically nonlinear parabolic PDEs - in particular, the incompressible Navier-Stokes equations. (For higher than quadratic 
nonlinearities, other approaches must be pursued Barrault et al. (2004); Cancès et al. (2007); Grepl et al. (2007a) that in turn introduce both numerical and theoretical complications.) Although there are many examples of reduced order models for the unsteady viscous Burgers equation Kunish and Volkwein (1999) and the unsteady incompressible Navier-Stokes equations Christensen et al. (2000); Deane et al. (1991); Gunzburger et al. (2007); Hinze and Volkwein (2005); Ito and Ravindran (1998a,b, 2001); Johansson et al. (2006), none is endowed with rigorous a posteriori error bounds. ${ }^{2}$ For the RB treatment of the viscous Burgers equation we refer to Nguyen et al. (2008).

For simplicity of exposition we consider the velocity formulation with homogeneous Dirichlet (no-slip) or periodic boundary conditions: thus the velocity space $Z$ is the space of all divergence-free functions $v$ in $\left(H^{1}(\Omega)\right)^{d=2}$ that vanish on all walls and are (say) $L$-periodic in $x_{1}$. We can then state the weak form of the incompressible Navier-Stokes equations (nondimensionalized with respect to the viscous scaling): for given $\mu \in \mathcal{D} \equiv\left[\mu_{\min }, \mu_{\max }\right]$ $\left(0<\mu_{\min } \leq \mu_{\max }\right)$, the velocity $u(t ; \mu)$ satisfies

$$
m\left(u_{t}(t ; \mu), v\right)+a(u(t ; \mu), v)+c(u(t ; \mu), u(t ; \mu), v)=\mu f(v), \quad \forall v \in Z, \forall t \in I,
$$

with initial condition $u(t=0 ; \mu), v=u_{0} \in Z$. We can subsequently evaluate our output of interest as $s(t ; \mu)=$ $\ell(u(t ; \mu))$. Our forms are given by $m(w, v) \equiv \int_{\Omega} w_{i} v_{i}, a(w, v) \equiv \int_{\Omega} w_{i, j} v_{i, j}$, and $c(w, z, v) \equiv \frac{1}{2} \int_{\Omega}\left(\left(w_{i} z_{j}\right)_{, j}+\right.$ $\left.z_{j} w_{i, j}\right) v_{i}$, where we adopt indicial notation; we presume that $f$ is a bounded functional over $X$, and $\ell$ is a bounded linear functional over $L^{2}(\Omega)$. Here $\mu$, our single parameter, denotes the magnitude of the driving force (not the dynamic viscosity - apologies); we then define our Reynolds number as

$$
\operatorname{Re}(\mu)=\frac{1}{L} \int_{\Omega} u_{1}\left(t_{f} ; \mu\right)
$$

which is similar to the "usual" channel definitions.

We next define the Euler-Backward discretization in time and the "truth" finite element approximation in space: Given divergence-free finite element spaces $Z^{\mathcal{N}} \subset Z$, we look for $u^{\mathcal{N} k}(\mu) \in Z^{\mathcal{N}}, 0 \leq k \leq K$, such that

$$
\frac{1}{\Delta t} m\left(u^{\mathcal{N} k}(\mu)-u^{\mathcal{N} k-1}(\mu), v\right)+a\left(u^{\mathcal{N} k}(\mu), v\right)+c\left(u^{\mathcal{N} k}(\mu), u^{\mathcal{N} k}(\mu), v\right)=\mu f(v), \quad \forall v \in Z^{\mathcal{N}},
$$

for $1 \leq k \leq K$, subject to initial condition $\left(u^{\mathcal{N} 0}(\mu), v\right)=\left(u_{0}, v\right), \forall v \in Z^{\mathcal{N}}$. We subsequently evaluate our output of interest as $s^{\mathcal{N} k}(\mu)=\ell\left(u^{\mathcal{N} k}(\mu)\right)$ for $0 \leq k \leq K$. We shall build our RB approximation upon the "truth" discretization (18), and we shall measure the error in our RB prediction relative to $u^{\mathcal{N} k}(\mu) \equiv u^{\mathcal{N}}\left(t^{k} ; \mu\right)$ and $s^{\mathcal{N} k}(\mu) \equiv s^{\mathcal{N}}\left(t^{k} ; \mu\right)$. For purposes of exposition, we consider the Euler-Backward scheme; the method is readily extended to the Crank-Nicolson discretization in time (as in our numerical results).

Lastly, we turn to the RB approximation. Our velocity space is given by $Z_{N} \equiv \operatorname{span}\left\{\xi_{n} \in Z^{\mathcal{N}}, 1 \leq n \leq N\right\}$; it immediately follows that $Z_{N} \subset Z^{\mathcal{N}}$, and we may hence pursue Galerkin projection with respect to (18). Given $\mu \in \mathcal{D}$, we look for $u_{N}^{k}(\mu) \in Z_{N}, 0 \leq k \leq K$, such that

$$
\frac{1}{\Delta t} m\left(u_{N}^{k}(\mu)-u_{N}^{k-1}(\mu), v\right)+a\left(u_{N}^{k}(\mu), v\right)+c\left(u_{N}^{k}(\mu), u_{N}^{k}(\mu), v\right)=\mu f(v), \quad \forall v \in Z_{N},
$$

for $1 \leq k \leq K$, subject to initial condition $u_{N}^{0}(\mu)=u^{\mathcal{N} 0}$. (For simplicity of exposition we assume that $u^{\mathcal{N} 0} \in$ $Z_{N}$.) We then evaluate our RB output as $s_{N}^{k}(\mu)=\ell\left(u_{N}^{k}(\mu)\right)$ for $0 \leq k \leq K$. (Clearly in proceeding with the divergence-free route we exclude outputs that depend on the pressure, as well as geometric parametrizations for which the incompressibility constraint is parameter dependent. Future work shall consider non-divergence-free spaces to address these issues and thus permit a wider class of applications.)

\subsubsection{A posteriori error estimation}

It can be shown by extension of the result in Nguyen et al. (2008) that the $L^{2}(\Omega)$-norm of the RB error, $\| u^{\mathcal{N} k}(\mu)-$ $u_{N}^{k}(\mu) \|$, can be bounded as

$$
\left\|u^{\mathcal{N} k}(\mu)-u_{N}^{k}(\mu)\right\| \leq \Delta_{N}^{k}(\mu), \quad 1 \leq k \leq K, \forall \mu \in \mathcal{D},
$$

where the error bound is defined (for $\Delta t$ sufficiently small Nguyen et al. (2008)) as

$$
\Delta_{N}^{k}(\mu)=\sqrt{\frac{\Delta t \sum_{k^{\prime}=1}^{k}\left(\varepsilon_{N}^{2}\left(t^{k^{\prime}} ; \mu\right) \prod_{j=1}^{k^{\prime}-1}\left(1+\Delta t \rho_{N}^{\mathrm{LB}}\left(t^{j} ; \mu\right)\right)\right)}{\prod_{k^{\prime}=1}^{k}\left(1+\Delta t \rho_{N}^{\mathrm{LB}}\left(t^{k^{\prime}} ; \mu\right)\right)}}, \quad 1 \leq k \leq K .
$$

\footnotetext{
${ }^{2}$ Note there are examples of rigorous RB a posteriori error bounds for the steady viscous Burgers equation in Veroy et al. (2003a) and the steady incompressible Navier-Stokes equations in Deparis (2008); Nguyen et al. (2005); Veroy and Patera (2005).
} 
Here $\varepsilon_{N}$ is the dual norm of the RB residual defined as

$$
r_{N}\left(v ; t^{k} ; \mu\right) \equiv \mu f(v)-\frac{1}{\Delta t} m\left(u_{N}^{k}(\mu)-u_{N}^{k-1}(\mu), v\right)-a\left(u_{N}^{k}(\mu), v\right)-c\left(u_{N}^{k}(\mu), u_{N}^{k}(\mu), v\right),
$$

and $\rho_{N}^{\mathrm{LB}}\left(t^{k} ; \mu\right)$ is a lower bound for the stability constant

$$
\rho_{N}\left(t^{k} ; \mu\right) \equiv \inf _{v \in Z^{\mathcal{N}}} \frac{2 c\left(u_{N}\left(t^{k} ; \mu\right), v, v\right)+a(v, v)}{\|v\|^{2}} .
$$

The output error bound can then be computed as $\Delta_{N}^{s k}(\mu)=\left(\sup _{v \in Z_{\mathcal{N}}} \ell(v) /\|v\|\right) \Delta_{N}^{k}(\mu)$ for $1 \leq k \leq K$.

The stability constant (23) is closely related to the absolute (immediate decay) criterion of hydrodynamic stability theory Joseph (1976). For $\mu$ sufficiently small (Reynolds $\operatorname{Re}(\mu)$ sufficiently small) $\rho_{N}(t ; \mu)$ will be uniformly positive and hence error growth will be controlled; in this case, we can consider rather large times effectively reaching steady or (say) steady-periodic states. However, for $\mu$ large (Reynolds $\operatorname{Re}(\mu)$ sufficiently large) $\rho_{N}(t ; \mu)$ will certainly be negative and hence the error bound (21) will grow exponentially in time; in this case, we will be practically limited to modest final times. The theory (e.g., a priori or even a posteriori finite element error estimates) for the Navier-Stokes equations Constantin and Foias (1988); Johnson et al. (1995) is plagued by exponential growth factors and large stability factors. There are some cases in which algebraic-in- $t_{f}$ bounds can be derived Johnson et al. (1995), however the requisite conditions will not always be satisfied.

The simplest and most common bounds for the exponential growth rate involve the $L^{\infty}(\Omega)$-norm of the gradient of the velocity (in our case, of the gradient of $u_{N}(t ; \mu)$ ) which indeed will increase with $\sqrt{\mathrm{Re}}$ or perhaps even Re. We believe our estimate (21),(23) will improve upon the usual theoretical estimates, not enough to permit long-time integration at very high Reynolds numbers, but enough to permit practical (and rigorous) error estimation at modest times and modest Reynolds numbers. There are two reasons for our optimism (in addition to some numerical results reported below): (23) includes a viscous stabilizing term that will somewhat constrain the minimizer and moderate the minimum - a candidate field large only in a thin destabilizing layer will also incur significant dissipation; $\rho_{N}(t ; \mu)$ of (23) shall be estimated (conservatively but) relatively precisely — our lower bound $\rho_{N}^{\mathrm{LB}}(t ; \mu)$ shall reflect the full temporal and spatial structure of the RB velocity field.

\subsubsection{Offline-Online computational approach}

We briefly describe the Offline-Online procedure for the incompressible Navier-Stokes equations and refer to Nguyen et al. (2008) for further details in the $d=1$ Burgers context. (Of course, the latter is not burdened with the incompressibility condition.)

\section{Construction-Evaluation decomposition}

For the Construction-Evaluation decomposition, the basic strategy remains intact — the dependence on $\mu$ is affine - however the procedure requires some modification from the linear case, and there is some degradation in performance. First, as regards $u_{N}(t ; \mu)$, the only new complication is the quadratic nonlinearity: the formation of the RB Jacobian matrix — required for Newton iteration of the implicit temporal discretization of (19) now requires $O\left(N^{3}\right)$ operations rather than $O\left(N^{2}\right)$; however, the total Evaluation operation count for $u_{N}(t ; \mu)$ and $s_{N}(t ; \mu)$ is relatively unchanged from the linear case. Second, as regards the error bound, (21), in particular the dual norm of the residual, there are two new complications: first, the presence of the quadratic nonlinearity increases the Evaluation operation count from $O\left(N^{2}\right)$ to $O\left(N^{4}\right)$ - certainly significant, but often not dominant (relative to the Newton iteration); second, the dual norm must be calculated with respect to the divergence-free space, and hence the Offline calculations — now essentially Stokes solves - are more complicated. However, and critically, the operation count for the Evaluation stage remains independent of $\mathcal{N}$.

We must also provide the lower bound $\rho_{N}^{\mathrm{LB}}(t ; \mu)$ for $\rho_{N}(t ; \mu)$ of $(23)$. To this end, we express $\rho_{N}\left(t^{k} ; \mu\right)$ as

$$
\rho_{N}\left(t^{k} ; \mu\right)=\min _{v \in Z^{\mathcal{N}}} \sum_{n=1}^{N+1} \Upsilon^{n}\left(t^{k} ; \mu\right) \frac{d^{n}(v, v)}{\|v\|^{2}} ;
$$

here $\Upsilon^{n}\left(t^{k} ; \mu\right)=\omega_{N n}\left(t^{k} ; \mu\right), 1 \leq n \leq N, \Upsilon^{N+1}\left(t^{k} ; \mu\right)=1$, and $d^{n}(w, v)=c\left(\xi_{n}, w, v\right)+c\left(\xi_{n}, v, w\right), 1 \leq n \leq$ $N, d^{N+1}(w, v)=a(w, v)$. We can thus apply the Successive Constraint Method (SCM) Huynh et al. (2007); Rozza et al. (to appear 2008) to implement the Construction-Evaluation decomposition for the lower bound $\rho_{N}^{\mathrm{LB}}(t ; \mu)$. The SCM is a general Offline-Online procedure for the calculation of a rigorous lower bound for 


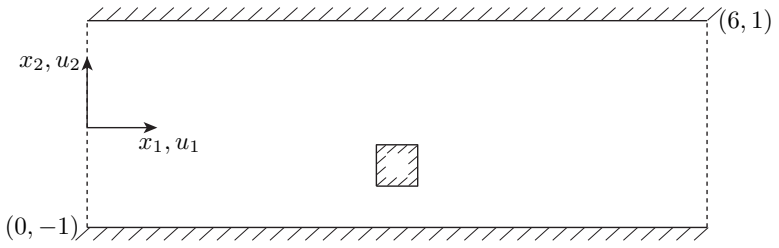

(a) geometry

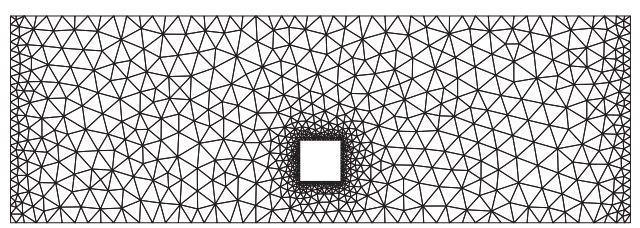

(b) mesh

Figure 1 The geometry (a) and mesh (b) for the square-in-channel configuration.

the minimum Rayleigh quotient of parametrically affine operators such as (24). (There are of course many simple techniques for either rigorous upper bounds or non-rigorous lower bounds for minimum eigenvalues or singular values; rigorous lower bounds are much more difficult to develop.)

In the SCM method the minimization (24) is recast as a Linear Progam: the linear objective is the Rayleigh quotient of (24) but now expressed in new variables $y^{n}=d^{n}(v, v) /\|v\|^{2}, 1 \leq n \leq N+1$; the linear inequality constraints reflect continuity information on the individual bilinear forms and stability information $\left(\operatorname{known} \rho_{N}\right)$ at optimally selected time and parameter values. The bound must be "useful," however high accuracy is not required; typically, an error of $50 \%$ in $\exp \left(\rho_{N}\left(t_{f} ; \mu\right) t_{f}\right)$ - ultimately a $2 \times$ degradation of our error bounds - is acceptable. The Construction stage, performed once, entails several (often many) eigenproblems over $Z^{\mathcal{N}}$ at $\operatorname{cost} O\left(\mathcal{N}^{*}\right)$. (In practice, the Offline SCM effort is often onerous; incompressible Navier-Stokes is particularly unpleasant since the div-free property of $Z^{\mathcal{N}}$ must be imposed through a Lagrange multiplier - yielding a Stokes eigenproblem.) The Evaluation/Online phase, performed for each desired $t^{k}, \mu$, is a small Linear Program of size independent of $\mathcal{N}$. The SCM contribution to the Online cost is quite small and often negligible.

\section{POD-Greedy sampling strategy}

The sampling procedure for Navier-Stokes is very similar to the POD-Greedy sampling procedure for linear parabolic equations described in Section 0.2.3. However, nonlinearity introduces several complications. First, we must calculate a nominal stability constant $\rho_{N}^{*}$ to serve (in lieu of $\rho_{N}^{\mathrm{LB}}$ ) in the POD-Greedy sampling procedure; then, once the sampling procedure is completed - and hence the RB approximation available - we (re)calculate the true stability constant. In the event that we find our nominal stability constant is not sufficiently conservative we can return to the sampling procedure to further refine the RB space. (Of course, in any case, in the Online stage - for any given $\mu$ of interest — we always calculate our rigorous a posteriori error bound (21) to confirm sufficient accuracy.) Second, since our problem is no longer Linear (-Time Invariant) we can no longer exploit the Impulse function as general "trainer": we must directly consider the (perhaps parametrized) control of interest.

\subsubsection{Numerical results}

We investigate two-dimensional incompressible flow in the square-in-channel configuration shown in Figure 1. The flow is assumed $L(=6)$-periodic in $x_{1}$. The channel domain (in our nondimensionalization) is thus of length $L=6$ and height $H=2$; the square obstacle, the bottom of which is located a distance $h=0.4$ above the bottom of the channel, is of side length $b=0.4$. The flow is driven by a pressure-gradient in the $x_{1}$-direction: $\mu f(v)=$ $\mu \int_{\Omega} v_{1}$. For our initial condition we choose the steady-state (stable) solution for $\mu=600, u^{\mathcal{N} 0}(\mu)=u^{\mathcal{N}}(t \rightarrow$ $\infty ; \mu=600)$; we integrate to a final time $t_{f}=0.5$. Our parameter domain is given by $\mathcal{D}=\left[\mu_{\min }=100, \mu_{\max }=\right.$ $1000]$; note that $\operatorname{Re}\left(\mu_{\min }\right)=40$ and $\operatorname{Re}\left(\mu_{\max }\right)=234$.

As might be expected from earlier investigations Karniadakis et al. (1988) in similar "eddy-promoter" geometries, the flow undergoes a supercritical Hopf bifurcation to a steady-periodic solution at $\mu=\mu_{\mathrm{cr}}$; for our particular geometry, $600<\mu_{\mathrm{cr}}<700$. Our choice $\mathcal{D}=\left[\mu_{\min }=100, \mu_{\max }=1000\right]$ hence captures the interesting dynamics. In particular, given any $\mu \in \mathcal{D}$, we simulate a "transition" or "response to disturbance": we expect oscillatory re-equilibration for $\mu<\mu_{\mathrm{cr}}$ and oscillatory growth (and ultimately nonlinear saturation) for $\mu>\mu_{\mathrm{cr}}$. Our final time $t_{f}=0.5$ is sufficiently large to clearly observe decay/growth — and in fact to almost reach a steady-state or steady-periodic solution even for $\mu=1000$. Note that $t_{f}=0.5$ in our viscous scaling actually corresponds to many convective timescales.

For the truth temporal discretization we take a Crank-Nicolson scheme with constant timestep $\Delta t=.001$ (in fact a relatively large Courant number) corresponding to $K=500$ time levels. (We note that Euler Backward 


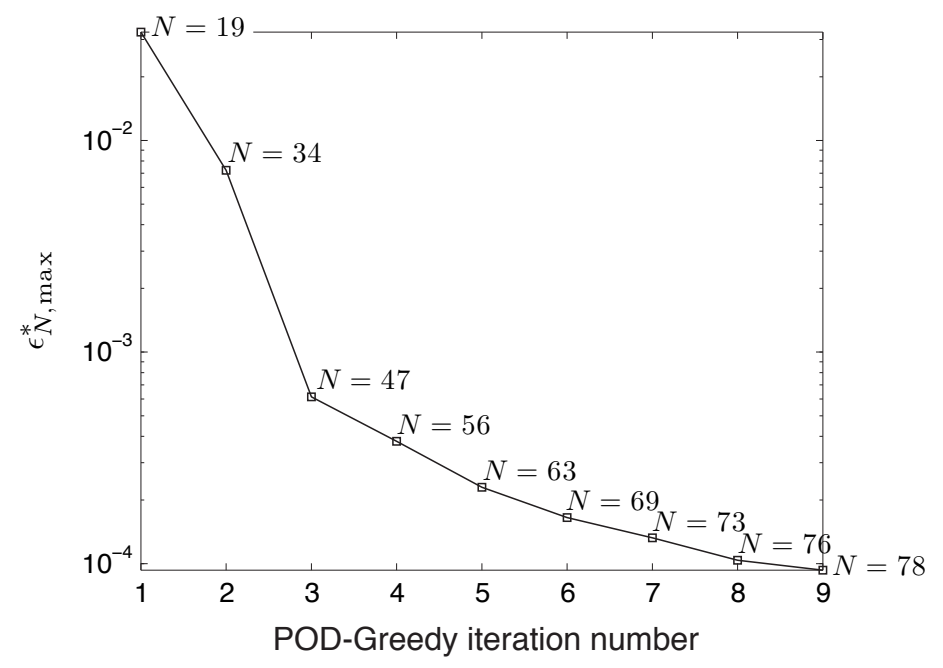

Figure 2 The error indicator $\epsilon_{N, \max }^{*}$ as a function of POD-Greedy iteration number and also $N$.

can only capture the correct bifurcation structure for very small $\Delta t(\ll .001)$ for which both the Offline and Online computational effort is prohibitively large.) We show in Figure 1(b) the "truth" FE triangulation; for the truth spatial discretization we take a classical $\mathbb{P}_{2}-\mathbb{P}_{1}$ (quadratic/linear) Taylor-Hood discretization Gunzburger (1989) with a total of $\mathcal{N}=7,361$ velocity and pressure degrees-of-freedom. Comparison of our truth solution with highly accurate spectral element calculations (Paul Fischer, private communication) confirms the validity of our "truth" results.

We next choose a $\log$ uniformly distributed sample $\Xi_{\text {train }} \subset \mathcal{D}$ of size $n_{\text {train }}=46$ and pursue the POD-Greedy sampling procedure with $\rho_{N}^{*}=0, \mu_{0}^{*}=1000$, and $\epsilon_{\mathrm{tol}}^{*}=10^{-4}$. The POD-Greedy sampling process terminates after 9 POD-greedy iterations - one iteration is defined as one pass through the while loop - and yields $N_{\max }=78$ and the optimal parameter sample $S^{*}=[1000,100,996,307,991,565,948,823,915]$. We observe, not surprisingly, that most of the POD-Greedy sample points are close to $\mu_{\max }=1000$, however lower $\mu$ are also represented. ${ }^{3}$ We present in Figure $2 \epsilon_{N, \max }^{*}$ as a function of POD-Greedy iteration number (as well as $N$ ). Clearly, the error indicator $\epsilon_{N, \max }^{*}$ decreases very rapidly with $N$; we shall subsequently confirm that the rigorous error bound, and hence also the true error, also decreases very rapidly with $N$.

We now turn to the stability factor. We perform the SCM procedure to construct the lower bound for the stability factor. We present in Figure 3 the stability factor $\rho_{N}\left(t^{k} ; \mu\right)$ as a function of $t^{k}$ for $\mu=400$ and $\mu=1000$ for $N=78$; we also present the stability factor lower bound $\rho_{N}^{\mathrm{LB}}\left(t^{k} ; \mu\right)$ as well as a corresponding upper bound $\rho_{N}^{\mathrm{UB}}\left(t^{k} ; \mu\right)$ (also provided by the $\mathrm{SCM}$ ). As already indicated, $\rho_{N}\left(t^{k} ; \mu\right)$ reflects viscous stabilization effects as well as the detailed spatial and temporal structure of the RB velocity field. For $\mu=400(\operatorname{Re}=110)-\mathrm{a}$ weakly nonlinear flow $-\rho_{N}\left(t^{k} ; \mu\right)$ increases with time $t^{k}$ and becomes positive (stable); for $\mu=1000(\operatorname{Re}=$ 234), a highly nonlinear flow, $\rho_{N}\left(t^{k} ; \mu\right)$ decreases with time $t^{k}$ and is negative (unstable) - but not too negative as measured in convective timescales. It should also be noted that the SCM method yields a very good (and significantly less complicated and less costly than a standard RB Rayleigh-Ritz approximation) upper bound for the stability factor: the difference between $\rho_{N}^{\mathrm{UB}}\left(t^{k} ; \mu\right)$ and $\rho_{N}\left(t^{k} ; \mu\right)$ is indeed very small. (If we replace $\rho_{N}^{\mathrm{LB}}\left(t^{k} ; \mu\right)$ with $\rho_{N}^{\mathrm{UB}}\left(t^{k} ; \mu\right)$ we will obtain better error bounds - but we can no longer provide guarantees.)

We present the vertical velocity at the spatial point $(3,0.24)$ as a function of time $t^{k}$ for both the "truth" FE and the $\mathrm{RB}$ approximation for $\mu=400(\mathrm{Re}=110)$ in Figure 4(a) and $\mu=1000(\mathrm{Re}=234$ ) in Figure 4(b). Despite the complex behavior of the flow and the relatively wide range of the effective Reynolds number, the RB approximation accurately captures the dynamics of the truth FE solution - re-equilibration below $\mu_{\mathrm{cr}}$ and oscillatory growth above $\mu_{\mathrm{cr}}$ - with only relatively few $(N=50)$ basis functions. We can attribute this rapid convergence to the Galerkin projection and the effectiveness of the POD-Greedy sampling procedure; the latter can be viewed as a systematic extension of earlier POD model reduction approaches Deane et al. (1991) applied, in fact, to "geometrically perturbed" channel flows very similar to our current square-in-channel configuration. Moreover,

\footnotetext{
${ }^{3}$ We choose to not make a second appeal to the POD-Greedy procedure once we obtain $\rho_{N}^{\mathrm{LB}}\left(t^{k} ; \mu\right), 1 \leq k \leq K$. In fact, since $\rho_{N}(\cdot ; \mu)$ decreases with $\mu$, the "min-max" POD-Greedy procedure based on the true $\rho_{N}^{\mathrm{LB}}\left(t^{k} ; \mu\right), 1 \leq k \leq K$, would further bias the sample $S^{*}$ towards $\mu=1000$.
} 


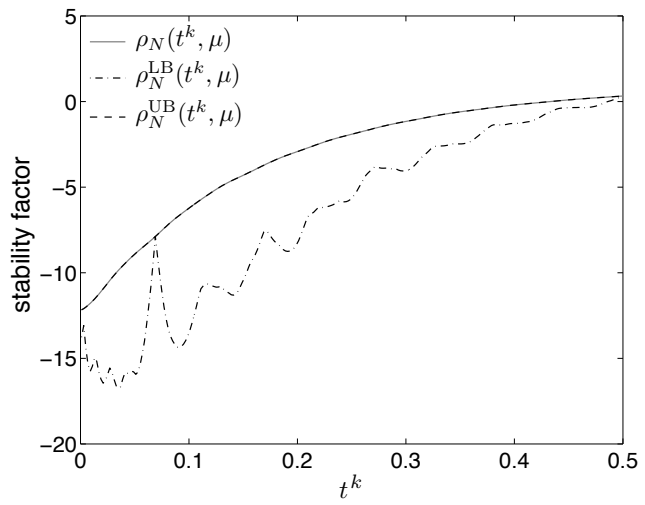

(a) $\mu=400$

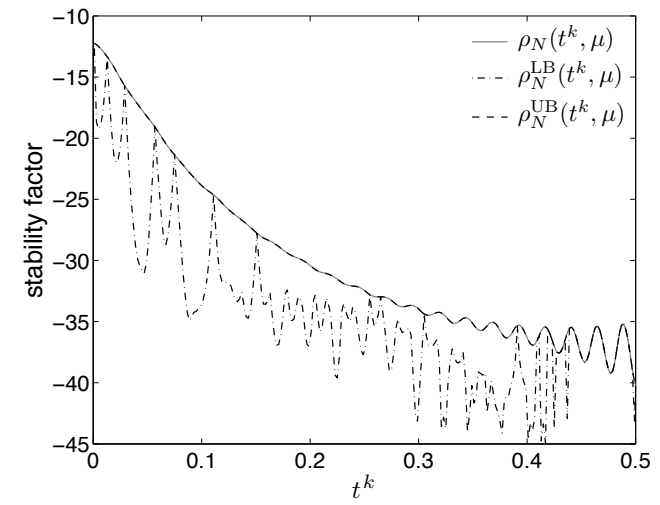

(b) $\mu=1000$

Figure 3 Stability factors $\rho_{N}\left(t^{k} ; \mu\right), \rho_{N}^{\mathrm{LB}}\left(t^{k} ; \mu\right)$, and $\rho_{N}^{\mathrm{UB}}\left(t^{k} ; \mu\right)$ as a function of $t^{k}$ for $N=78$ : (a) $\mu=400$, and (b) $\mu=1000$.

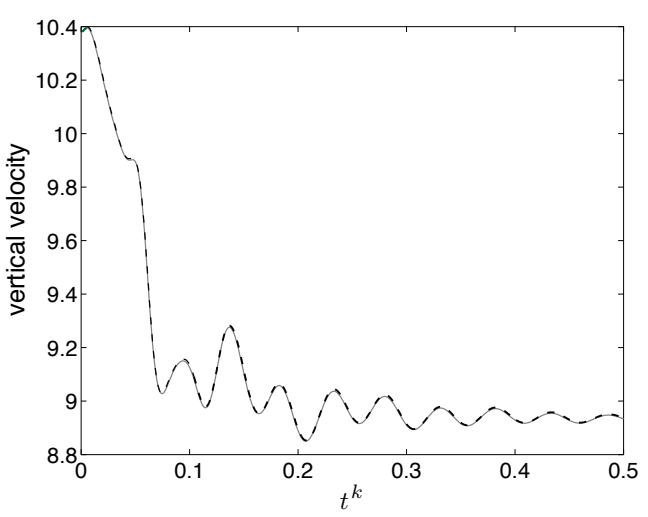

(a) $\mu=400$

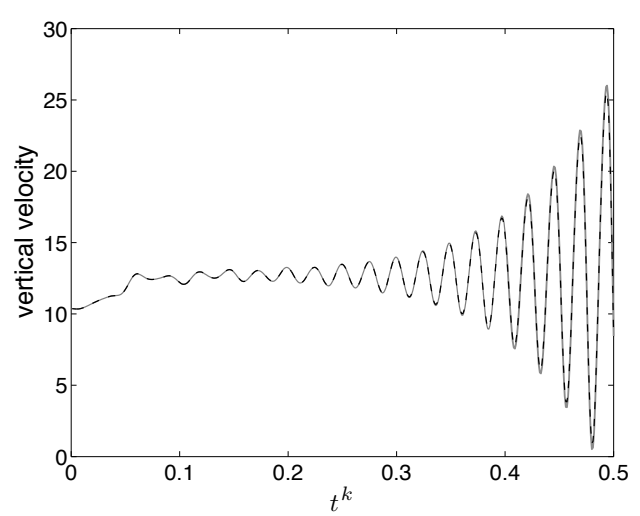

(b) $\mu=1000$

Figure 4 Comparison between the FE (gray solid line) and RB (dashed line) solutions at the spatial point $(3,0.24)$ as a function of time $t^{k}$ for $N=50$ : (a) $\mu=400$ and (b) $\mu=1000$.

calculation of the RB output $s_{N}^{k}(\mu), 1 \leq k \leq K$, in the Online stage is $2,400 \times$ faster than direct evaluation of the FE output $s^{\mathcal{N} k}(\mu), 1 \leq k \leq K$. (It is possible that with more efficient FE Navier-Stokes solvers the savings would be reduced from $O(1000)$ to $O(100)$ — but still quite significant.)

Finally, and perhaps most importantly, we present in Figure 5 the true relative $L^{2}(\Omega)$ FE-RB error, $\| u^{\mathcal{N} k}(\mu)-$ $u_{N}^{k}(\mu)\|/\| u^{\mathcal{N} k}(\mu) \|$, and the relative $L^{2}(\Omega)$ FE-RB error bound, $\Delta_{N}\left(t^{k} ; \mu\right) /\left\|u^{\mathcal{N} k}(\mu)\right\|$, as a function of discrete time $t^{k}$ for $N=30,45,60$. We consider the particular case $\mu=1000(\operatorname{Re}=234)$ : similar results are obtained for all $\mu \in[100,1000]$; in fact, and in particular given our single appeal to the POD-Greedy procedure, the errors true and a posteriori bound - are largest for $\mu=1000$. We observe that both the true error and the a posteriori error bound do in fact converge quite rapidly with $N$. We also observe that both the true error and the a posteriori error bound do indeed grow exponentially in time, as might be expected for a supercritical linear instability. (Of course, whereas the true error will saturate, the error bound will not saturate, and hence in the unsteady context we must in practice limit the final time $t_{f}$; we discuss this further below.) Finally, the effectivity — the ratio of the error bound to the true error — is not too large.

It is crucial to note from Figure 5 that, even for our moderate final time and "large" Reynolds number $(\operatorname{Re}=$ $234)$, the error bound is still quite meaningful. In particular, from Online evaluation of $\Delta_{N=60}\left(t_{f} ; \mu=1000\right)$ we can guarantee that the $(N=60) \mathrm{FE}-\mathrm{RB}$ error in the relative $L^{2}(\Omega)$ norm $^{4}$ is no greater than $2.6 \%$ for all $t \in\left[0, t_{f}\right]$. Furthermore, the RB Online calculation is very inexpensive: $u_{N}^{k}(\mu)$ and $\Delta_{N}^{k}(\mu), 1 \leq k \leq K$, as well as the output

\footnotetext{
${ }^{4}$ Of course in practice to compute Online the relative $L^{2}(\Omega)$ norm error bound we conservatively replace the denominator $\left\|u^{\mathcal{N} k}(\mu)\right\|$ with the very inexpensive surrogate $\left\|u_{N}^{k}(\mu)\right\|-\Delta_{N}^{k}(\mu)$.
} 


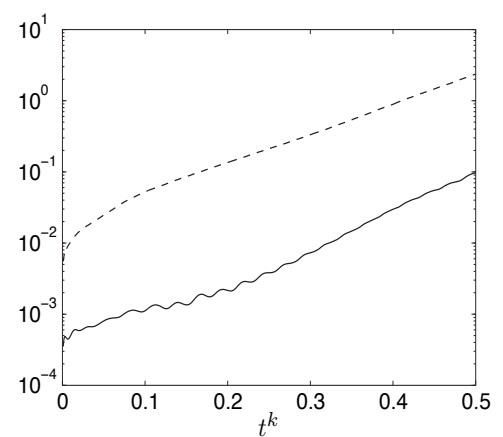

(a) $N=30$

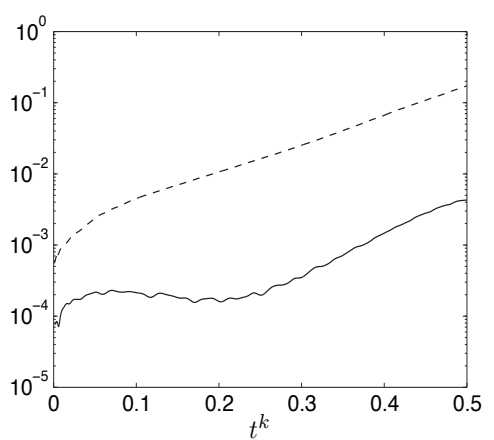

(b) $N=45$

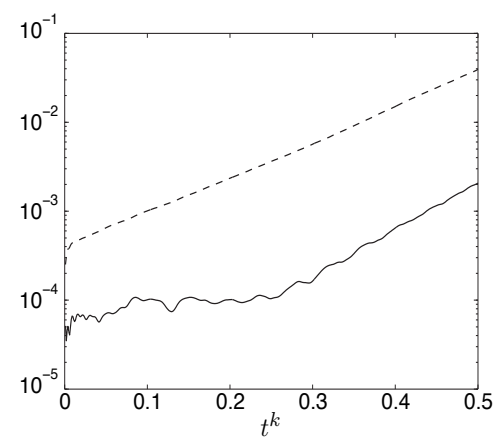

(c) $N=60$

Figure 5 The true relative $L^{2}(\Omega)$ error, $\left\|u^{\mathcal{N} k}(\mu)-u_{N}^{k}(\mu)\right\| /\left\|u^{\mathcal{N} k}(\mu)\right\|$ (solid line), and the relative $L^{2}(\Omega)$ error bound, $\Delta_{N}^{k}(\mu) /\left\|u^{\mathcal{N} k}(\mu)\right\|$ (dashed line), for $\mu=1000$ as a function of discrete time $t^{k}$ for $N=30,45$, and 60 .

of interest $s_{N}^{k}(\mu)$ (e.g., flowrate) and associated a posteriori output error bound $\Delta_{N}^{s k}(\mu)=\|\ell\|_{L^{2}(\Omega)} \Delta_{N}^{k}(\mu), 1 \leq$ $k \leq K$, can be computed very rapidly - roughly 52 seconds on a Pentium IV $1.73 \mathrm{GHz}$ processor compared to over 4 hours for direct FE evaluation of $u^{\mathcal{N} k}(\mu), s^{\mathcal{N} k}(\mu), 1 \leq k \leq K$.

Much additional effort is required to consider more extensive (geometry and force) parametrizations, more complicated boundary conditions, and more general (velocity and pressure) outputs. And we will never be able to consider very large times for very high Reynolds numbers although more advanced techniques, such as adjoint methods Johnson et al. (1995); Pierce and Giles (2000) should extend our reach. Nevertheless, our example illustrates that we can indeed consider modest final times and modest Reynolds numbers with significant nonlinear effects. There are many interesting applications in this "attainable" region of Reynolds number-final time space.

\subsection{Bayesian Parameter Estimation}

\subsubsection{Bayesian approach}

In parameter estimation problems we would like to infer the unknown parameter $\mu^{*} \in \mathcal{D} \subset \mathbb{R}^{P}$ from the measurements of outputs of interest, $s^{(m)}\left(t ; \mu^{*}\right), 1 \leq m \leq M$, collected for $t=t^{k_{j}^{\exp }}=k_{j}^{\exp } \Delta t \in\left[0, t_{f}\right], 1 \leq j \leq J$; here $M$ is the number of outputs and $J$ is the number of measurements per output. (In actual practice, some of the $P$ parameters - for example, measurement system design variables — may be specified (or optimized) rather than inferred.) In our case the outputs are expressed as functionals of the solution of the forward problem (1) $s^{(m)}\left(t ; \mu^{*}\right)=\ell^{(m)}\left(u\left(t ; \mu^{*}\right)\right)$ for $1 \leq m \leq M$. In order to assess our approach to parameter estimation we create "synthetic" data as

$$
G_{m j}^{\exp }\left(\mu^{*} ; \varepsilon_{\exp }\right)=s^{(m) \mathcal{N}}\left(t_{j}^{k_{j}^{\exp }} ; \mu^{*}\right)+\varepsilon_{m j}^{\exp }, \quad 1 \leq m \leq M, 1 \leq j \leq J,
$$

where the $s^{(m) \mathcal{N}}\left(t^{k_{j}^{\text {exp }}} ; \mu^{*}\right)$ are the "truth" FE approximation to the exact output $s^{(m)}\left(t^{k_{j}^{\exp }} ; \mu^{*}\right)$ and the $\varepsilon_{m j}^{\exp }$ represent the "experimental" error. We assume the $\varepsilon_{m j}^{\exp }$ to be independent identically distributed (i.i.d.) Gaussian random variables (hence white in time) with zero mean and known variance $\sigma_{\text {exp }}^{2}$.

We apply the Bayesian approach to parameter estimation Mosegaard and Tarantola (2002); Wang and Zabaras (2005a) to the FE "truth" discretization of the forward problem (1). The expected value $\mathrm{E}^{\mathcal{N}}\left[\mu^{*} \mid G^{\exp }\right]$ of the unknown parameter $\mu^{*}$ conditional on the data $G^{\text {exp }}$ is given by

$$
\mathrm{E}^{\mathcal{N}}\left[\mu^{*} \mid G^{\exp }\right]=\frac{\int_{\mathcal{D}} \mu \Pi^{\mathcal{N}}\left(G^{\exp } \mid \mu\right) \Pi_{0}(\mu) d \mu}{\int_{\mathcal{D}} \Pi^{\mathcal{N}}\left(G^{\exp } \mid \mu^{\prime}\right) \Pi_{0}\left(\mu^{\prime}\right) d \mu^{\prime}} .
$$

Here the likelihood function $\Pi^{\mathcal{N}}\left(G^{\exp } \mid \mu\right)$ is given by

$$
\Pi^{\mathcal{N}}\left(G^{\exp } \mid \mu\right)=\left(\frac{1}{2 \pi \sigma_{\exp }^{2}}\right)^{M J / 2} \exp \left(-\frac{\left(G^{\exp }-F^{\mathcal{N}}(\mu)\right)^{T}\left(G^{\exp }-F^{\mathcal{N}}(\mu)\right)}{2 \sigma_{\exp }^{2}}\right),
$$

${ }^{5}$ For brevity we consider only the expectation; our methodology also applies to the variance and indeed the full empirical posterior distribution function. 
where, for $1 \leq m \leq M$ and $1 \leq j \leq J, F_{m j}^{\mathcal{N}}: \mu \in \mathcal{D} \rightarrow s^{(m) \mathcal{N}}\left(t^{k_{j}^{\exp }} ; \mu\right)$ denotes the FE evaluation of the $m^{\text {th }}$ output at time $t_{j}^{k_{j}^{\exp }}$ at any given $\mu$ in our parameter domain $\mathcal{D}$. The prior distribution on the parameter $\mu, \Pi_{0}(\mu)$, is also assumed Gaussian 6

$$
\Pi_{0}(\mu)=\left(\frac{1}{2 \pi \sigma_{0}^{2}}\right)^{P / 2} \exp \left(-\frac{\left(\mu-\mu_{0}\right)^{T}\left(\mu-\mu_{0}\right)}{2 \sigma_{0}^{2}}\right)
$$

where $\mu_{0} \in \mathcal{D}$ is the prior mean and $\sigma_{0}^{2}$ is the associated variance (more generally a covariance). Note that $\mathrm{E}^{\mathcal{N}}\left[\mu^{*} \mid G^{\exp }\right]$ in (26) is an expectation with respect to the "random" parameter $\mu$ : for any given measurement, $G^{\exp }, \mathrm{E}^{\mathcal{N}}\left[\mu^{*} \mid G^{\exp }\right]$ is our estimator for $\mu^{*}$; properly speaking, $\mathrm{E}^{\mathcal{N}}\left[\mu^{*} \mid G^{\exp }\right]$ is a realization of a random variable - a function of $G^{\exp }$. (To avoid cumbersome notation, $G^{\exp }$ refers both to the measurement random variable and to associated realizations.)

The expected value in (26) necessitates the computation of multidimensional integrals, which in turn require numerous evaluations of the FE outputs; as a consequence, the parameter estimation procedure can be very expensive. To reduce the computational cost of Bayesian inverse analysis Wang and Zabaras (2005b) introduce POD-based model reduction. Our emphasis here is a posteriori error estimation (absent in earlier Bayesian model reduction approaches): our error bounds ensure that our Bayesian inferences are $(i)$ certifiably accurate (relative to the FE truth), and (ii) as efficient as possible — through optimal choice of $N$ for a given error tolerance. In the subsequent subsection, we incorporate our a posteriori error bounds into the Bayesian approach to permit rapid and reliable parameter estimation. (See also Grepl (2005); Grepl et al. (2007b); Nguyen (2008a) for an alternative approach to RB inverse analysis which more explicitly characterizes parameter uncertainty.)

\subsubsection{A posteriori bounds for the expected value}

We develop here inexpensive, rigorous lower and upper bounds for the expected value (26) based on the RB outputs and associated error bounds. Toward this end, we first introduce $F_{N m j}(\mu)=s_{N}^{(m)}\left(t_{j}^{k_{j}^{\exp }} ; \mu\right)$ and $\Delta F_{N m j}(\mu)=$ $\Delta_{N}^{s(m)}\left(t_{j}^{k_{j}^{\exp }} ; \mu\right)$ for $1 \leq m \leq M$ and $1 \leq j \leq J$, and then $F_{N}^{ \pm}(\mu)=F_{N}(\mu) \pm \Delta F_{N}(\mu)$. Here $s_{N}^{(m)}\left(t^{k} ; \mu\right)$ and $\Delta_{N}^{s(m)}\left(t^{k} ; \mu\right)$ are the RB prediction and associated error bound for the $m^{\text {th }}$ output. We then define, for $1 \leq m \leq$ $M$ and $1 \leq j \leq J, B_{N m j}(\mu)=\max \left\{\left|G_{m j}^{\exp }-F_{N m j}^{-}(\mu)\right|,\left|G_{m j}^{\exp }-F_{N m j}^{+}(\mu)\right|\right\}$, and

$$
A_{N m j}(\mu)= \begin{cases}0, & \text { if } G_{m j}^{\exp } \in\left[F_{N m j}^{-}(\mu), F_{N m j}^{+}(\mu)\right], \\ \min \left\{\left|G_{m j}^{\exp }-F_{N}^{-}(\mu)\right|,\left|G_{m j}^{\exp }-F_{N}^{+}(\mu)\right|\right\}, & \text { otherwise } .\end{cases}
$$

Note that $G^{\exp } \in \mathbb{R}^{M J}, F_{N}^{ \pm}(\mu) \in \mathbb{R}^{M J}, A_{N}(\mu) \in \mathbb{R}^{M J}$, and $B_{N}(\mu) \in \mathbb{R}^{M J}$.

We now introduce two new likelihood functions

$$
\begin{aligned}
& \Pi_{N}^{a}\left(G^{\exp } \mid \mu\right)=\left(\frac{1}{2 \pi \sigma_{\exp }^{2}}\right)^{M J / 2} \exp \left(-\frac{A_{N}^{T}(\mu) A_{N}(\mu)}{2 \sigma_{\exp }^{2}}\right), \\
& \Pi_{N}^{b}\left(G^{\exp } \mid \mu\right)=\left(\frac{1}{2 \pi \sigma_{\exp }^{2}}\right)^{M J / 2} \exp \left(-\frac{B_{N}^{T}(\mu) B_{N}(\mu)}{2 \sigma_{\exp }^{2}}\right),
\end{aligned}
$$

from which we may evaluate

$$
\mathrm{E}_{N}^{\mathrm{LB}}\left[\mu^{*} \mid G^{\exp }\right]=\frac{\int_{\mathcal{D}} \mu \Pi_{N}^{b}\left(G^{\exp } \mid \mu\right) \Pi_{0}(\mu) d \mu}{\int_{\mathcal{D}} \Pi_{N}^{a}\left(G^{\exp } \mid \mu^{\prime}\right) \Pi_{0}\left(\mu^{\prime}\right) d \mu^{\prime}}, \quad \mathrm{E}_{N}^{\mathrm{UB}}\left[\mu^{*} \mid G^{\exp }\right]=\frac{\int_{\mathcal{D}} \mu \Pi_{N}^{a}\left(G^{\exp } \mid \mu\right) \Pi_{0}(\mu) d \mu}{\int_{\mathcal{D}} \Pi_{N}^{b}\left(G^{\exp } \mid \mu^{\prime}\right) \Pi_{0}\left(\mu^{\prime}\right) d \mu^{\prime}} .
$$

(If $\mu$ takes on negative values then (31) must be modified slightly.) We shall take $\mathrm{E}_{N}^{\mathrm{AV}}\left[\mu^{*} \mid G^{\exp }\right]=\frac{1}{2}\left(\mathrm{E}_{N}^{\mathrm{LB}}\left[\mu^{*} \mid G^{\exp }\right]+\right.$ $\left.\mathrm{E}_{N}^{\mathrm{UB}}\left[\mu^{*} \mid G^{\exp }\right]\right)$ as our RB approximation to $\mathrm{E}^{\mathcal{N}}\left[\mu^{*} \mid G^{\exp }\right]$.

It can be shown that the expected values defined in (31) satisfy

$$
\mathrm{E}_{N}^{\mathrm{LB}}\left[\mu^{*} \mid G^{\exp }\right] \leq \mathrm{E}^{\mathcal{N}}\left[\mu^{*} \mid G^{\exp }\right] \leq \mathrm{E}_{N}^{\mathrm{UB}}\left[\mu^{*} \mid G^{\exp }\right],
$$

and hence $\left|\mathrm{E}^{\mathcal{N}}\left[\mu^{*} \mid G^{\exp }\right]-\mathrm{E}_{N}^{\mathrm{AV}}\left[\mu^{*} \mid G^{\exp }\right]\right| \leq \frac{1}{2} \Delta \mathrm{E}_{N}\left[\mu^{*} \mid G^{\exp }\right] \equiv \frac{1}{2}\left(\mathrm{E}_{N}^{\mathrm{UB}}\left[\mu^{*} \mid G^{\exp }\right]-\mathrm{E}_{N}^{\mathrm{LB}}\left[\mu^{*} \mid G^{\exp }\right]\right)$. The proof is simple: we first note that, since $\left|s^{(m) \mathcal{N}}\left(t^{k_{j}^{\exp }} ; \mu\right)-s_{N}^{(m)}\left(t^{k_{j}^{\exp }} ; \mu\right)\right| \leq \Delta_{N}^{s(m)}\left(t^{k_{j}^{\exp }} ; \mu\right)$,

$$
F_{N}^{-}(\mu) \leq F^{\mathcal{N}}(\mu) \leq F_{N}^{+}(\mu), \quad \forall \mu \in \mathcal{D}
$$

${ }^{6}$ In theory, we must multiply (28) by a pre-factor reflecting the bounded $\mathcal{D}$. In practice, we shall consider small $\sigma_{0}$ and large $\mu_{0}$ such that $\mu$ outside $\mathcal{D}$ are highly improbable — and hence $\mathcal{D}$ is effectively $\mathbb{R}^{P}$. 
it thus follows that

$$
A_{N}(\mu)^{T} A_{N}(\mu) \leq\left(G^{\exp }-F^{\mathcal{N}}(\mu)\right)^{T}\left(G^{\exp }-F^{\mathcal{N}}(\mu)\right) \leq B_{N}(\mu)^{T} B_{N}(\mu),
$$

and hence

$$
\Pi_{N}^{b}\left(G^{\exp } \mid \mu\right) \leq \Pi^{\mathcal{N}}\left(G^{\exp } \mid \mu\right) \leq \Pi_{N}^{a}\left(G^{\exp } \mid \mu\right) .
$$

The bound result (32) is a direct consequence of the definitions (31) and inequality (35), and the non-negativity of $\Pi^{a}, \Pi^{b}, \Pi_{0}$, and (here) $\mu \in \mathcal{D}$.

In actual practice the integrations of (31) are replaced with a numerical quadrature; in fact, our FE-RB bounds are still rigorous for any quadrature scheme (e.g., Gauss or Monte Carlo) with positive weights. In this paper we consider an adaptive piecewise Gauss-Legendre technique: we first create a domain decomposition selectively refined near an approximate $\mu^{*}$; we then apply standard tensor-product Gauss-Legendre quadrature within each subdomain. We denote by $n_{\text {quad }}$ the total number of integrand evaluations required. (Note for given $G^{\exp }$ the RB outputs and associated error bounds are computed (only once) and stored on the quadrature grid; we can then evaluate the several requisite integrals without further appeal to the RB approximation.) For problems with more parameters, Monte Carlo techniques would be necessary.

In the Offline stage the RB is constructed: the POD-Greedy sampling procedure is invoked and all necessary Online quantities are computed and stored. Then, in the Online stage (which involves only the Evaluation phase), for each new identification $\left(\mu^{*}\right)$ - and hence for each new $G^{\exp }$ provided - we evaluate in "real-time" the expectation lower and upper bounds (31). It is clear that the RB approach will be much faster than direct FE evaluation (of the requisite integrals) even for a single identification, and even more efficient for multiple identifications: in the limit that $n_{\text {quad }}$ and/or the number of identifications tends to infinity, the RB Offline effort is negligible - only the very fast $(\mathcal{N}$-independent) RB Online evaluations are relevant. Equivalently, if our emphasis is on real-time identification, again only the very fast RB Online evaluations are important.

\subsubsection{Numerical example}

We consider the application of transient thermal analysis to detection of flaws/defects in a Fiber-Reinforced Polymer (FRP) composite bonded to a concrete (C) slab Grepl (2005); Starnes (2002). Since debonds or delaminations at the composite-concrete interface often occur (even at installation), effective and real-time quality control providing reliable information about the thickness and fiber content of the composite, and the location and size of defects - is vital to safety.

We show the FRP-concrete system in Figure 6. The FRP layer is of thickness $h_{\mathrm{FRP}}$ and (truncated) lateral extent $10 h_{\mathrm{FRP}}$; the concrete layer is of (truncated) depth and lateral extent $5 h_{\mathrm{FRP}}$ and $10 h_{\mathrm{FRP}}$, respectively. We presume that a delamination crack of unknown length $w_{\text {del }}$ centered at $x_{1}=0$ is present at the FRP-concrete interface. The FRP thermal conductivity, specific heat, and density are given by $k, c$, and $\rho$ with subscripts FRP and $\mathrm{C}$, respectively. We shall assume that the FRP and concrete share the same known values for both the density and specific heat. We assume that the FRP (respectively, concrete) conductivity is unknown (respectively, known); we denote the (unknown) conductivity ratio as $\kappa=k_{\mathrm{FRP}} / k_{\mathrm{C}}$. (In practice, the FRP conductivity depends on fiber orientation and content - and hence somewhat unpredictable.)

We nondimensionalize all lengths by $h_{\mathrm{FRP}} / 2$ and all times by $h_{\mathrm{FRP}}^{2} \rho_{C} c_{C} / k_{C}$. The nondimensional temperature $u$ is given by $\left(T-T_{0}\right) /\left(T_{\mathrm{FRP}, \max }-T_{0}\right)$, where $T$ is the dimensional temperature, $T_{0}$ is the initial temperature (uniform in both the FRP and concrete), and $T_{\mathrm{FRP}, \max }$ is the maximum allowable FRP temperature. The nondimensional flux - imposed at the FRP exposed surface, as shown in Figure $6-g(t)$ is given by $q(t) h_{\mathrm{FRP}} /\left(2 k_{C}\left(T_{\mathrm{FRP}, \max }-T_{0}\right)\right)$, where $q(t)$ is the dimensional flux. We presume that the nondimensional surface heat flux $g(t)$ - the stimulus — is unity for $0 \leq t \leq 5$ and zero for all $t>5$.

Upon application of our mapping procedures (to a reference domain with crack length $w_{\text {del }}=3$ ) Rozza et al. (to appear 2008) we arrive at the problem statement (1) with affine expansions (2) for $Q_{a}=15, Q_{m}=2$. (In fact, due to symmetry, we consider only half the domain: $x_{1}>0$.) Our initial condition is $u=0$; we integrate to a final time $t_{f}=10.0$. Our $P=2$ (both "unknown") parameters are $\mu \equiv\left(\mu_{1}, \mu_{2}\right) \equiv\left(w_{\mathrm{del}} / 2, \kappa\right)$ assumed to reside in the parameter domain $\mathcal{D} \equiv[1,5] \times] 0.5,2[$. Finally, we introduce our truth discretization: we consider Euler backward discretization in time with $\Delta t=0.05$ and hence $K=200$ time levels $t^{k}=k \Delta t, 0 \leq k \leq K$; we consider a linear FE truth approximation space $X^{\mathcal{N}}$ of dimension $\mathcal{N}=3581$. (The FE triangulation provides high resolution in the vicinity of the surface and near the crack tip, the two regions which suffer sharp spatial gradients.)

Finally, we consider $M=2$ outputs: as shown in Figure 6, each output functional corresponds to the average of the (temperature) field over a "small" square of side-length 1 (flush with the exposed FRP surface); the square for the first output is centered at (measurement site 1) $x_{1}=0$, while the square for the second output is centered 


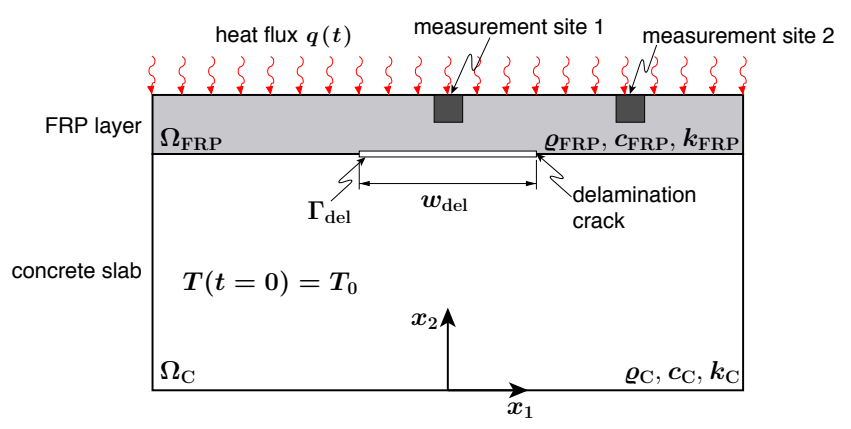

Figure 6 Delamination of a FRP layer bonded to a concrete slab.

Table 1 Lower bound, upper bound, and bound gap for the expected value of the delamination half-width $\mu_{1}$ and conductivity ratio $\mu_{2}$ as a function of $N$. The true parameter value is $\mu_{1}^{*}=2.8$ and $\mu_{2}^{*}=0.9$.

\begin{tabular}{ccccccc}
\hline & \multicolumn{3}{c}{ Delamination half-width } & \multicolumn{3}{c}{ Conductivity ratio } \\
\hline$N$ & $\mathrm{E}_{N}^{\mathrm{LB}}\left[\mu_{1}^{*}\right]$ & $\mathrm{E}_{N}^{\mathrm{UB}}\left[\mu_{1}^{*}\right]$ & $\Delta \mathrm{E}_{N}\left[\mu_{1}^{*}\right]$ & $\mathrm{E}_{N}^{\mathrm{LB}}\left[\mu_{2}^{*}\right]$ & $\mathrm{E}_{N}^{\mathrm{UB}}\left[\mu_{2}^{*}\right]$ & $\Delta \mathrm{E}_{N}\left[\mu_{2}^{*}\right]$ \\
\hline 10 & 1.8199 & 4.5112 & 2.6912 & 0.5748 & 1.4251 & 0.8503 \\
20 & 2.6646 & 3.0698 & 0.4052 & 0.8415 & 0.9695 & 0.1280 \\
30 & 2.8302 & 2.8890 & 0.0588 & 0.8948 & 0.9134 & 0.0186 \\
40 & 2.8495 & 2.8702 & 0.0207 & 0.9010 & 0.9076 & 0.0066 \\
\hline
\end{tabular}

at (measurement site 2) $x_{1}=6.5$. Note that we must consider a small area average (rather than pointwise measurement) to ensure that our output functionals remain bounded over $L^{2}(\Omega)$ (indeed, even over $H^{1}(\Omega)$; the $L^{2}(\Omega)$ norm of these "area averaging" functionals increases as the inverse of the square root of the area.

We first briefly discuss the RB approximation and error bounds, and then turn to the inverse problem. This PDE is not too difficult: we need an RB space of dimension only $N=40$ to ensure - based on $\Delta_{N}^{s(m)}\left(t^{k}, \mu\right), m=$ 1,2 - a "certified" accuracy of roughly $1 \%$ in both outputs. ${ }^{7}$ For $N=40$ the Online RB calculation $\mu \rightarrow$ $s_{N}^{(m)}\left(t^{k} ; \mu\right), \Delta_{N}^{s(m)}\left(t^{k} ; \mu\right), 0 \leq k \leq K$, is effected in 0.16 seconds; in constrast, direct FE evaluation requires 22 seconds. All computations in this section are carried out on a $1.73 \mathrm{GHz}$ Pentium IV processor with $1 \mathrm{~GB}$ memory.

We now turn to parameter estimation. We focus on the sensitivity of the parameter estimation procedure to the RB dimension $N$ as (inexpensive but rigorously) quantified by our expectation error bounds. In this experiment, we set $\mu^{*}=\left(\mu_{1}^{*}, \mu_{2}^{*}\right)=\left(w_{\mathrm{del}}^{*}, \kappa^{*}\right)=(2.8,0.9)$ and $\sigma_{\exp }^{2}=0.0025$; we choose for the prior mean and variance $\mu_{0}=(3.3,1.2)$ and $\sigma_{0}^{2}=0.04$, respectively. The synthetic experimental data (25) is generated by adding i.i.d. Gaussian random variables to our $M=2$ outputs evaluated at $J=20$ time levels $t^{k_{j}^{\text {exp }}}, k_{j}^{\text {exp }}=10 j, 1 \leq j \leq J$. We then apply our adaptive piecewise Gauss-Legendre quadrature algorithm with $n_{\text {quad }}=10,000$ points. We present in Table 1 the lower bound, $\mathrm{E}_{N}^{\mathrm{LB}}\left[\mu_{p}^{*}\right]$, upper bound, $\mathrm{E}_{N}^{\mathrm{UB}}\left[\mu_{p}^{*}\right]$, and bound gap $\Delta \mathrm{E}_{N}\left[\mu_{p}^{*}\right], p=1,2$, for the expected value of the unknown parameter $\mu^{*}$; we consider a single realization $G^{\exp }$.

We observe that the bound gaps $\Delta \mathrm{E}_{N}\left[\mu_{p}^{*}\right]=\mathrm{E}_{N}^{\mathrm{UB}}\left[\mu_{p}^{*}\right]-\mathrm{E}_{N}^{\mathrm{LB}}\left[\mu_{p}\right], p=1,2$, decrease rapidly: as $N$ increases, $\Delta_{N}^{s(m)}\left(t^{k} ; \mu\right) \rightarrow 0$ and hence $A_{N}(\mu) \rightarrow B_{N}(\mu)$ rapidly. The parameter estimator is quite accurate: the expectation bounds are within the white noise $(5.0 \%)$ of the true parameter value $\mu^{*}=(2.8,0.9)$, biased toward $\mu_{0}$ as expected. The RB Online computation (for $N=40$ ) of the lower and upper bounds for the expected value is completed in approximately 27 minutes — arguably "real-time" for this particular application — as opposed to 61 hours for direct FE evaluation. The RB Offline time is roughly only 2.4 hours, and hence even for one identification the RB approach "pays off"; for several identifications, the RB Offline effort will be negligible. (If real-time response "in the field" is imperative, then even for one identification the RB Offline effort is not important.) In short, we are guaranteed the fidelity of the truth FE approximation but at the cost of a low order model.

\footnotetext{
${ }^{7}$ In fact, the effectivity — the ratio of the output error bound to the true output error — is rather large, $O(100)$, and hence the actual accuracy for $N=40$ is closer to $10^{-4}$. However, since in the Online stage our inferences are based on the (inexpensive) error bound, we must construct an RB approximation for which the error bound is sufficiently accurate.
} 


\section{Bibliography}

Almroth BO, Stern P and Brogan FA 1978 Automatic choice of global shape functions in structural analysis. AIAA Journal 16, 525-528.

Balmes E 1996 Parametric families of reduced finite element models: Theory and applications. Mechanical Systems and Signal Processing 10(4), 381-394.

Barrault M, Y.Maday, Nguyen NC and Patera AT 2004 An "empirical interpolation" method: Application to efficient reducedbasis discretization of partial differential equations. C. R. Acad. Sci. Paris, Série I. 339, 667-672.

Boyaval S 2008 Reduced-basis approach for homogenization beyond the periodic setting. SIAM Multiscale Modeling \& Simulation 7(1), 466-494.

Boyaval S, Bris CL, Nguyen NC and Patera AT 2008 A reduced-basis approach for variational problems with stochastic parameters: Application to heat conduction with variable robin coefficient. Comp. Meth. Appl. Mech. and Eng.

Bui-Thanh T, Willcox K and Ghattas O 2007 Model reduction for large-scale systems with high-dimensional parametric input space (AIAA Paper 2007-2049) Proceedings of the 48th AIAA/ASME/ASCE/AHS/ASC Structures, Structural Dynamics and Material Conference.

Cancès E, Le Bris C, Nguyen NC, Maday Y, Patera AT and Pau GSH 2007 Feasibility and competitiveness of a reduced basis approach for rapid electronic structure calculations in quantum chemistry Proceedings of the Workshop for Highdimensional Partial Differential Equations in Science and Engineering (Montreal).

Christensen EA, Brons M and Sorensen JN 2000 Evaluation of pod-based decomposition techniques applied to parameterdependent non-turbulent flows. SIAM J. Sci. Comput. 21, 1419-1434.

Constantin P and Foias C 1988 Navier-Stokes Equations Chicago Lectures in Mathematics. University of Chicago Press, Chicago, IL.

Deane A, Kevrekidis I, Karniadakis G and Orszag S 1991 Low-dimensional models for complex geometry flows: Application to grooved channels and circular cylinders. Phys. Fluids 10, 2337-2354.

Deparis S 2008 Reduced basis error bound computation of parameter-dependent Navier-Stokes equations by the natural norm approach. SIAM Journal of Numerical Analysis 46, 2039-2067.

Fink JP and Rheinboldt WC 1983 On the error behavior of the reduced basis technique for nonlinear finite element approximations. Z. Angew. Math. Mech. 63(1), 21-28.

Grepl M 2005 Reduced-Basis Approximations and A Posteriori Error Estimation for Parabolic Partial Differential Equations $\mathrm{PhD}$ thesis Massachusetts Institute of Technology.

Grepl MA and Patera AT 2005 A Posteriori error bounds for reduced-basis approximations of parametrized parabolic partial differential equations. M2AN (Math. Model. Numer. Anal.) 39(1), 157-181.

Grepl MA, Maday Y, Nguyen NC and Patera AT 2007a Efficient reduced-basis treatment of nonaffine and nonlinear partial differential equations. M2AN (Math. Model. Numer. Anal.) 41, 575-605.

Grepl MA, Nguyen NC, Veroy K, Patera AT and Liu GR 2007b Certified rapid solution of partial differential equations for realtime parameter estimation and optimization In Proceedings of the $2^{\text {nd }}$ Sandia Workshop of PDE-Constrained Optimization: Real-Time PDE-Constrained Optimization (ed. Biegler LT, Ghattas O, Heinkenschloss M, Keyes D and van B. Wandeers B), pp. 197-216 SIAM Computational Science and Engineering Book Series.

Gunzburger MD 1989 Finite Element Methods for Viscous Incompressible Flows. Academic Press.

Gunzburger MD, Peterson J and Shadid JN 2007 Reduced-order modeling of time-dependent PDEs with multiple parameters in the boundary data. Comp. Meth. Applied Mech. 196, 1030-1047.

Haasdonk B and Ohlberger M 2008 Reduced basis method for finite volume approximations of parametrized evolution equations. Mathematical Modelling and Numerical Analysis (M2AN) 42(2), 277-302.

Hinze M and Volkwein S 2005 Proper orthogonal decomposition surrogate models for nonlinear dynamical systems: error estimates and suboptimal control Lecture Notes in Computational Science and Engineering vol. 45 Springer.

Huynh DBP, Rozza G, Sen S and Patera AT 2007 A successive constraint linear optimization method for lower bounds of parametric coercivity and inf-sup stability constants. C. R. Acad. Sci. Paris, Analyse Numérique 345(8), 473-478.

Ito K and Ravindran SS 1998a A reduced basis method for control problems governed by PDEs In Control and Estimation of Distributed Parameter Systems (ed. Desch W, Kappel F and Kunisch K) Birkhäuser pp. 153-168.

Ito K and Ravindran SS 1998b A reduced-order method for simulation and control of fluid flows. Journal of Computational Physics 143(2), 403-425. 
Ito K and Ravindran SS 2001 Reduced basis method for optimal control of unsteady viscous flows. International Journal of Computational Fluid Dynamics 15(2), 97-113.

Johansson PS, Andersson H and Rønquist E 2006 Reduced-basis modeling of turbulent plane channel flow. Computers and Fluids 35(2), 189-207.

Johnson C, Rannacher R and Boman M 1995 Numerical and hydrodynamic stability: Towards error control in computational fluid dynamics. SIAM Journal of Numerical Analysis 32(4), 1058-1079.

Joseph D 1976 Stability of fluid motions. I. \& II. vol. 27 \& 28 of Springer Tracts in Natural Philosophy. Springer-Verlag, New York.

Karniadakis GE, Mikic BB and Patera AT 1988 Minimum-dissipation transport enhancement by flow destabilization - reynolds' analogy revisited. Journal of Fluid Mechanics 192, 365-391.

Kunisch K and Volkwein S 2002 Galerkin proper orthogonal decomposition methods for a general equation in fluid dynamics. SIAM J. Num. Analysis 40(2), 492-515.

Kunish K and Volkwein S 1999 Control of burgers' equation by a reduced order approach using proper orthogonal decomposition. J. Optimization Theory and Applications 102, 345-371.

Machiels L, Maday Y, Oliveira IB, Patera A and Rovas D 2000 Output bounds for reduced-basis approximations of symmetric positive definite eigenvalue problems. C. R. Acad. Sci. Paris, Série I 331(2), 153-158.

Mosegaard K and Tarantola A 2002 Probabilistic approach to inverse problems. International Handbook of Earthquake and Engineering Seismology, Part A pp. 237-265.

Nguyen NC 2008a Efficient and robust parameter estimation using the reduced basis method: Application to the inverse scattering problem. SIAM J. Sci. Comput.

Nguyen NC 2008b A multiscale reduced-basis method for parametrized elliptic partial differential equations with multiple scales. Journal of Computational Physics. To Appear.

Nguyen NC, Rozza G and Patera AT 2008 Reduced basis approximation and a posteriori error estimation for the timedependent viscous burgers equation. Calcolo.

Nguyen NC, Veroy K and Patera AT 2005 Certified real-time solution of parametrized partial differential equations In Handbook of Materials Modeling (ed. Yip S) Springer pp. 1523-1558.

Noor AK and Peters JM 1980 Reduced basis technique for nonlinear analysis of structures. AIAA Journal 18(4), 455-462.

Pierce N and Giles MB 2000 Adjoint recovery of superconvergent functionals from PDE approximations. SIAM Review 42(2), 247-264.

Porsching TA 1985 Estimation of the error in the reduced basis method solution of nonlinear equations. Mathematics of Computation 45(172), 487-496.

Prud'homme C, Rovas D, Veroy K, Maday Y, Patera A and Turinici G 2002 Reliable real-time solution of parametrized partial differential equations: Reduced-basis output bounds methods. Journal of Fluids Engineering 124(1), 70-80.

Quarteroni A and Valli A 1997 Numerical Approximation of Partial Differential Equations 2nd edn. Springer.

Rozza G, Huynh DBP and Patera AT (to appear) 2008 Reduced basis approximation and a posteriori error estimation for affinely parametrized elliptic coercive partial differential equations: Application to transport and continuum mechanics. Archives Computational Methods in Engineering. Available online at doi: 10.1007/s11831-008-9019-9.

Starnes M 2002 Development of Technical Bases for Using Infrared Thermography for Nondestructive Evaluation of Fiber Reinforced Polymer Composites Bonded to Concrete PhD thesis Massachusetts Institute of Technology.

Veroy K and Patera AT 2005 Certified real-time solution of the parametrized steady incompressible Navier-Stokes equations; Rigorous reduced-basis a posteriori error bounds. International Journal for Numerical Methods in Fluids 47, 773-788.

Veroy K, Prud'homme C and Patera AT 2003a Reduced-basis approximation of the viscous Burgers equation: Rigorous $a$ posteriori error bounds. C. R. Acad. Sci. Paris, Série I 337(9), 619-624.

Veroy K, Prud'homme C, Rovas DV and Patera AT 2003b A Posteriori error bounds for reduced-basis approximation of parametrized noncoercive and nonlinear elliptic partial differential equations Proceedings of the 16th AIAA Computational Fluid Dynamics Conference. Paper 2003-3847.

Wang J and Zabaras N 2005a Hierarchical bayesian models for inverse problems in heat conduction. Inverse Problems 21 , 183-206.

Wang J and Zabaras N 2005b Using bayesian statistics in the estimation of heat source in radiation. International Journal of Heat and Mass Transfer 48, 15-29. 